\title{
Multi-resolution flow simulations by smoothed particle hydrodynamics via domain decomposition
}

\author{
Xin Bian, Zhen Li, George Em Karniadakis* \\ Division of Applied Mathematics, Brown University, Providence, RI 02912, USA
}

\begin{abstract}
We present a methodology to concurrently couple particle-based methods via a domain decomposition (DD) technique for simulating viscous flows. In particular, we select two resolutions of the smoothed particle hydrodynamics (SPH) method as demonstration. Within the DD framework, a simulation domain is decomposed into two (or more) overlapping sub-domains, each of which has an individual particle scale determined by the local flow physics. Consistency of the two sub-domains is achieved in the overlap region by matching the two independent simulations based on Lagrangian interpolation of state variables and fluxes. The domain decomposition based SPH method (DD-SPH) employs different spatial and temporal resolutions, and hence, each sub-domain has its own smoothing length and time step. As a consequence, particle refinement and derefinement are performed asynchronously according to individual time advancement of each sub-domain. The proposed strategy avoids SPH force interactions between different resolutions on purpose, so that coupling, in principle, can go beyond SPH $\mathrm{SPH}$, and may allow SPH to be coupled with other mesoscopic or microscopic particle methods.

The DD-SPH method is validated first for a transient Couette flow, where simulation results based on proper coupling of spatial-temporal scales agree well with analytical solutions. In particular, we find that the size of the overlap region should be at least $r_{c, 1}+2 r_{c, 2}$, where $r_{c, 1}$ and $r_{c, 2}$ are cut off radii in the two sub-domains with $r_{c, 1} \leq r_{c, 2}$. Subsequently, a perturbation wave is considered traveling either parallel or perpendicular to the hybrid interface. Compressibility is significant if transient behavior at short sonic-time-scale is relevant, while the fluid can be treated as quasi-incompressible at sufficiently long time scale. To this end, we propose a coupling of density fields from the two sub-domains. Finally, a steady Wannier flow is simulated, where a rotating cylinder is placed next to a wall. Lubrication effects are prominent in the gap between the cylinder and the bottom wall, rendering a high resolution necessary, whereas in the rest of the domain the flow can be simulated at much lower resolution. DD-SPH simulation results with both spatial and temporal resolution ratios up to 16 agree well with the results of a single high resolution simulation, but with the former two-orders of magnitude faster in the region away from the cylinder.
\end{abstract}

Keywords: multiscale coupling, temporal coupling, hybrid simulation, Lagrangian particle methods, mesoscopic modeling 


\section{Introduction}

In the last few decades there have been many efforts to develop multiscale methods for fluid flows. There are mainly two types of multiscale methods. The first method adopts the same fluid solver over the whole computational domain and applies high resolution at locations with high gradients. Hence, it is essentially a multi-resolution method, for example, the adaptive mesh refinement (AMR) [1] method belongs to this category. The second method couples heterogeneous solvers applied in different regions of the computational domain. For example, a Navier-Stokes (NS) solver is applied in one part of the domain, while a molecular dynamics (MD) method is applied in the other part of the domain where the microscopic boundary condition is unknown a priori $[2,3,4,5]$. In our work, we aim to formulate a more general framework for Lagrangian particle methods, which can accomplish both types of multiscale simulation. To this end, we adopt the domain decomposition (DD) methodology [6], which has been primarily utilized in coupling heterogeneous solvers. Here, we apply the DD methodology on adaptively refining the smoothed particle hydrodynamics (SPH) method [7]. The DD methodology exhibits both flexibility and computational efficiency when applied in a multi-resolution simulation.

In contrast to the mesh-based discretizations, such as the AMR, multi-resolution in particle-based discretizations seems to be more difficult to deal with due to its Lagrangian nature. However, it is also exactly the Lagrangian property that provides particle-based methods with advantages for certain applications, such as advection dominated problems, multiphase flows with complex boundaries [8,9], and free surface flows [7], among others. Therefore, it is tempting to develop a multi-resolution scheme in particle-based discretization of fluid flows. In fact, there have been already several innovative efforts. For example, in an astrophysical application of SPH method, a variable smoothing length was adopted to account for changes in particle number density so that the dynamic range of spatial resolution is increased [10]. In another engineering application of SPH method, a dynamic procedure of particle refinement was proposed, where a parent particle is split into a couple of daughter particles to increase local numerical accuracy [11]. Based on the particle refinement procedure, a de-refinement algorithm was developed to merge a few small particles into a large one so that computational cost may be saved [12]. It is worth noting that these works target the spatial multi-resolution, but the temporal resolution is not distinguished between small and large particles. Since these schemes are formulated based on SPH force interactions between different spatial resolutions, forces must be exchanged instantaneously between particles of various sizes. This instant exchange is the key cause of the difficulty of separating temporal advancement of different spatial resolutions.

To enable decoupling on both spatial and temporal resolutions, we propose to couple state variables and fluxes between SPH simulations of different resolutions. In particular, we decompose a global domain into two (or possibly more) overlapping sub-domains, each of which performs a SPH simulation with independent spatial and

\footnotetext{
${ }^{*}$ Corresponding author

Email addresses: xin_bian@brown.edu (Xin Bian), zhen_li@brown.edu (Zhen Li), george_karanidakis@brown. edu (George Em Karniadakis)
} 
temporal resolution according to the local flow physics. A hybrid description exists in the overlap region, and consistency of the two simulations is achieved by constraining each simulation's artificial boundary, which is embedded in the other sub-domain. The constraint is performed on state variables by a Lagrangian interpolation based on information of the other simulation. The state variables are density and momentum for the isothermal fluid considered here. In addition, we introduce a simple and effective conditional rule to correct the pressure force calculation with a non-spherical support at the artificial boundary, which resolves automatically also the density inconsistency in the same region. A particle is removed once it leaves the sub-domain while a new particle is inserted in the artificial boundary, under the guidance of fluxes in the other simulation. Since SPH blending-force interactions between two types of particles are intentionally avoided, both smoothing length and time step are separated for the two simulations. This implies that particle deletion and insertion are asynchronous in the two sub-domains and are performed according to the local time advancement. Synchronization of two simulations is done at selective time instants. Therefore, the new coupling scheme enables both spatial and temporal speed-ups in the low resolution sub-domain.

The remainder of this paper is organized as follows. In Section 2, we briefly review the Lagrangian hydrodynamic equations and the corresponding discrete SPH equations. Later in the same section, we introduce the separation of two particle number densities on two sub-domains based on the domain decomposition method. The associated artificial boundary condition for particle methods and particle deletion/insertion procedure are also explained in detail. In Section 3, we validate the coupling of momentum and density of two simulations, and the density/pressure wave across the overlap region with hybrid description, by performing simulations of transient Couette flow and wave perturbations. Furthermore, we quantify the numerical error on temporal coupling with different communication time steps between two simulations. We also examine the accuracy on the algorithms for asynchronous particle deletion and insertion for the Wannier flow problem with fluxes across hybrid interface. In Section 4, we summarize this work and suggest possible extensions.

\section{The multiscale particle model based on domain decomposition}

\subsection{Lagrangian hydrodynamic equations}

We consider an isothermal Newtonian fluid with a Lagrangian description governed by the continuity and the Navier-Stokes equations as follows [13]:

$$
\begin{aligned}
\frac{d \rho}{d t} & =-\rho \nabla \cdot \mathbf{v} \\
\rho \frac{d \mathbf{v}}{d t} & =-\nabla p+\eta \nabla^{2} \mathbf{v}+\frac{\eta}{3} \nabla \nabla \cdot \mathbf{v},
\end{aligned}
$$

where $\rho, \mathbf{v}, p$, and $\eta$ are material density, velocity, pressure and shear viscosity, respectively. An equation of state (EOS) relating pressure to density is needed to provide a closure for the weakly compressible description. Both an ideal-gas-like linear EOS and 
liquid-like stiff EOS are widely used in the literature [7, 14]:

$$
\begin{aligned}
& p=c_{s}^{2} \rho, \\
& p=\frac{c_{s}^{2} \rho_{e q}}{7}\left[\left(\frac{\rho}{\rho_{e q}}\right)^{7}-1\right]+\chi,
\end{aligned}
$$

where $\rho_{e q}$ is the equilibrium density. The artificial sound speed $c_{s}$ is chosen based on a scale analysis $[7,14]$ such that the pressure field reacts strongly to small deviations in density and quasi-incompressibility is fulfilled. Here, $\chi$ is a positive constant to enforce non-negativity of pressure on any SPH particle [8]. In principle, the constant $\chi$ under the gradient operator in Eq. (2) should have no contribution to the momentum balance. We shall illustrate the performance of Eqs.(3a) and (3b) for a weakly compressible flow in Section 3.

\subsection{Smoothed particle hydrodynamics}

For a comprehensive description of smoothed particle hydrodynamics (SPH) method we refer to recent review articles [7, 15]. Here we adopt one classical SPH formulation with a B-spline kernel applied to viscous flows [14]. However, the proposed coupling methodology should apply to other SPH formulations [7] or other SPH kernels, such as the Wendland functions [16].

For convenience, as reference we define some simple notations

$$
\begin{aligned}
\mathbf{r}_{i j} & =\mathbf{r}_{i}-\mathbf{r}_{j}, \\
\mathbf{v}_{i j} & =\mathbf{v}_{i}-\mathbf{v}_{j}, \\
\mathbf{e}_{i j} & =\mathbf{r}_{i j} / r_{i j}, \quad r_{i j}=\left|\mathbf{r}_{i j}\right|,
\end{aligned}
$$

where $\mathbf{r}_{i}, \mathbf{v}_{i}$ are position and velocity of particle $i ; \mathbf{r}_{i j}, \mathbf{v}_{i j}$ are relative position and velocity of particles $i$ and $j$; $r_{i j}$ is the distance of the two and $\mathbf{e}_{i j}$ is the unit vector pointing $j$ to $i$. The equation of motion for SPH particles and the corresponding discrete continuity and momentum equations are:

$$
\begin{aligned}
\dot{\mathbf{r}}_{i} & =\mathbf{v}_{i}, \\
d_{i} & =\frac{\rho_{i}}{m_{i}}=\sum_{j} W\left(r_{i j}\right)=\sum_{j} W_{i j}, \\
m_{i} \dot{\mathbf{v}}_{i} & =\sum_{j \neq i}\left(\mathbf{F}_{i j}^{C}+\mathbf{F}_{i j}^{D}\right),
\end{aligned}
$$

where $d$ is particle number density defined as the ratio of density $\rho$ and particle mass $m$ (constant), and it is calculated based on neighboring particle positions. $W(r)$ is a bell-shaped weighting function named as SPH kernel and it has at least two properties

$$
\lim _{h \rightarrow 0} W\left(\mathbf{r}-\mathbf{r}^{\prime}, h\right)=\delta\left(\mathbf{r}-\mathbf{r}^{\prime}\right), \quad \int W\left(\mathbf{r}-\mathbf{r}^{\prime}, h\right) d \mathbf{r}^{\prime}=1,
$$

where $h$ is defined as smoothing length. Any kernel adopted should converge to the Dirac delta function $\delta$ as $h \rightarrow 0$ and its integral must be normalized. In this work a 
high-order quintic kernel from the B-spline family is adopted, which has been widely utilized since the inception of SPH simulation for viscous flows [14]:

$$
W(s)=c_{D}\left\{\begin{array}{lr}
(3-s)^{5}-6(2-s)^{5}+15(1-s)^{5}, & 0 \leq s<1 \\
(3-s)^{5}-6(2-s)^{5}, & 1 \leq s<2 \\
(3-s)^{5}, & 2 \leq s<3 \\
0, & s \geq 3
\end{array}\right.
$$

where $s=3 r_{i j} / r_{c}=r_{i j} / h$ and $r_{c}$ is a finite cut off radius based on user's choice to limit numerical errors. The normalization coefficients are $c_{2}=63 /\left(478 \pi r_{c}{ }^{2}\right)$ and $c_{3}=$ $81 /\left(360 \pi r_{c}{ }^{3}\right)$ in two and three dimensions, respectively.

Note that Eqs. (5) and (6) already account for the continuity equation Eq. (1), which does not need to be discretized explicitly. $\mathbf{F}_{i j}^{C}$ and $\mathbf{F}_{i j}^{D}$ are pairwise conservative and dissipative forces between particles, corresponding to a discretization of forces due to pressure and viscous stress in Navier-Stokes equations. Following a thermodynamic framework, an expression for conservative force is obtained as follows [17],

$$
\mathbf{F}_{i j}^{C}=-\left(\frac{P_{i}}{d_{i}^{2}}+\frac{P_{j}}{d_{j}^{2}}\right) \frac{\partial W}{\partial r_{i j}} \mathbf{e}_{i j}
$$

which was also previously derived in a mathematical manner and has been widely utilized [7]. $\mathbf{F}_{i j}^{C}=-\mathbf{F}_{j i}^{C}$ (Newton's 3rd law) and they are active along $\mathbf{e}_{i j}$, therefore both linear and angular momenta are strictly conserved. The second-order derivatives in Eq. (2), corresponding to the viscous forces on particle $i$, can be discretized as [17],

$$
\begin{aligned}
\frac{1}{d_{i}}\left(\nabla^{2} \mathbf{v}\right)_{i} & =2 \sum_{j} \frac{1}{d_{i} d_{j} r_{i j}} \frac{\partial W}{\partial r_{i j}} \mathbf{v}_{i j}, \\
\frac{1}{d_{i}}(\nabla \nabla \cdot \mathbf{v})_{i} & =\sum_{j} \frac{1}{d_{i} d_{j} r_{i j}} \frac{\partial W}{\partial r_{i j}}\left[(D+2) \mathbf{e}_{i j} \cdot \mathbf{v}_{i j} \mathbf{e}_{i j}-\mathbf{v}_{i j}\right],
\end{aligned}
$$

where the dimension $D(=2$ or 3$)$ of the problem is explicitly introduced into the expression. Therefore, the pairwise dissipative force is

$$
\mathbf{F}_{i j}^{D}=\frac{\eta}{d_{i} d_{j} r_{i j}} \frac{\partial W}{\partial r_{i j}}\left(\frac{2 D-1}{D} \mathbf{v}_{i j}+\frac{D+2}{D} \mathbf{e}_{i j} \cdot \mathbf{v}_{i j} \mathbf{e}_{i j}\right) .
$$

$\mathbf{F}_{i j}^{D}$ also satisfies Newton's 3rd law so that it conserves linear momentum.

We adopt the velocity Verlet integrator for the time integration of SPH particles. To maintain numerical stability of the explicit scheme, the time-step size $\Delta t$ is restricted by two conditions: $\Delta t \leq 0.25 h / c_{s}$ and $\Delta t \leq 0.125 h^{2} / v$ (kinematic viscosity $v=\eta / \rho$ ). For low Reynolds number flows, the viscous constraint with a squared dependence on $h$ usually dominates.

\subsection{Domain decomposition method}

We consider a domain decomposition (DD) method based on the classical alternating Schwartz scheme [6]. A computational domain has two external boundaries defined 


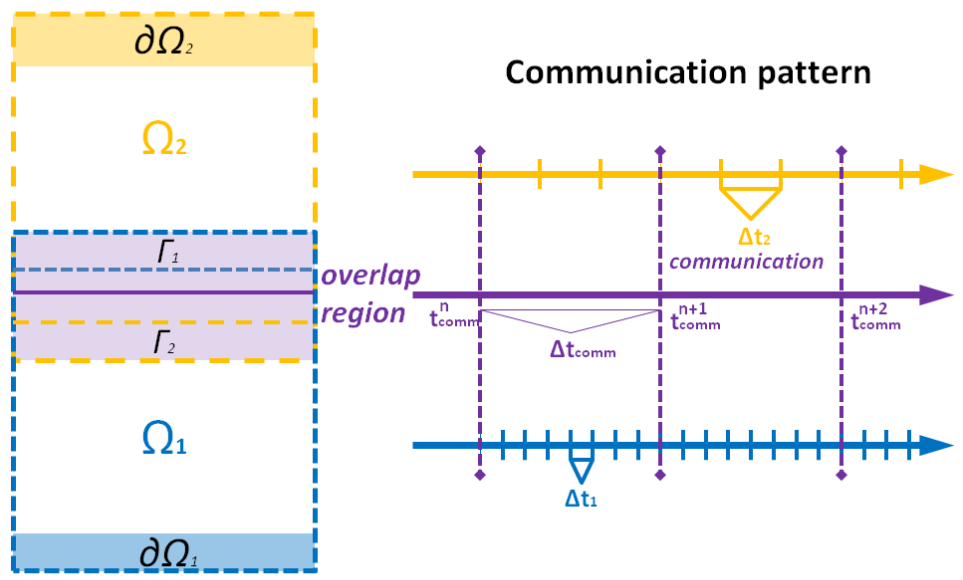

Figure 1: Sketch of domain decomposition (left) and communication pattern (right). The shadowed region is the overlap region of the two sub-domains. $\partial \Omega_{1}$ and $\partial \Omega_{2}$ are external boundaries while $\Gamma_{1}$ and $\Gamma_{2}$ are artificial boundaries. Sub-domain $\Omega_{1}$ has time step $\Delta t_{1}$ and sub-domain $\Omega_{2}$ has time step $\Delta t_{2}$. Values in $\Gamma_{1}$ and $\Gamma_{2}$ are updated every $\Delta t_{\text {comm }}$.

in $\partial \Omega_{1}$ and $\partial \Omega_{2}$ and periodic boundaries in other directions. The global domain is decomposed into two (or more) overlapping sub-domains, as sketched in Fig. 1. An artificial boundary $\Gamma_{1}$ is attached to $\Omega_{1}$ and it is embedded in $\Omega_{2}$; likewise, $\Gamma_{2}$ is an artificial boundary for $\Omega_{2}$ and it is embedded in $\Omega_{1}$. Therefore, the bottom sub-domain consists of $\Omega_{1} \cup \partial \Omega_{1} \cup \Gamma_{1}$, while the top sub-domain is made of $\Omega_{2} \cup \partial \Omega_{2} \cup \Gamma_{2}$.

For a steady problem, a simple alternating Schwartz method together with an iterative solver (e.g., Jacobi method) starts with an initial guess value of $\omega_{1}^{0}$ for $\Omega_{1}$ and $\omega_{2}^{0}$ for $\Omega_{2}$ at 0 th step. Subsequently, it solves the problem in sub-domain $\Omega_{1}$ with two Dirichlet boundary conditions in $\partial \Omega_{1}$ and $\Gamma_{1}$, to obtain $\omega_{1}^{1}$ as an updated value in $\Omega_{1}$. Values in $\Gamma_{1}$ are nothing else but a sub-set of $\omega_{2}^{0}$ in $\Omega_{2}$. Concurrently, it solves the problem in sub-domain $\Omega_{2}$ with two Dirichlet boundary conditions in $\partial \Omega_{2}$ and $\Gamma_{2}$ to obtain $\omega_{2}^{1}$ as an updated value in $\Omega_{2}$. Values in $\Gamma_{2}$ are a sub-set of $\omega_{1}^{0}$ in $\Omega_{1}$. This alternating procedure repeats until the two solutions in the overlap region (defined as $\Gamma_{1}, \Gamma_{2}$ and the gap region between them) match within a certain tolerance. Exchange of artificial boundary values takes place every $\Delta t_{\text {comm }}$ steps. The time step here is a pseudo-time for relaxation if we deal with a steady problem.

For a linear system, the procedure just described is equivalent to a block Jacobi method. We obtain new solutions at two sub-domains concurrently, based on the values of each other at last step. In contrast, the original alternating Schwartz method is identical to the block Gauss-Seidel method and solves two sub-domains consecutively. Therefore, it utilizes updated values of the other sub-domain and has better convergence [6]. We prefer the block-Jacobi-type of alternating procedure in this work, however, by considering its advantages in the context of parallel execution. 


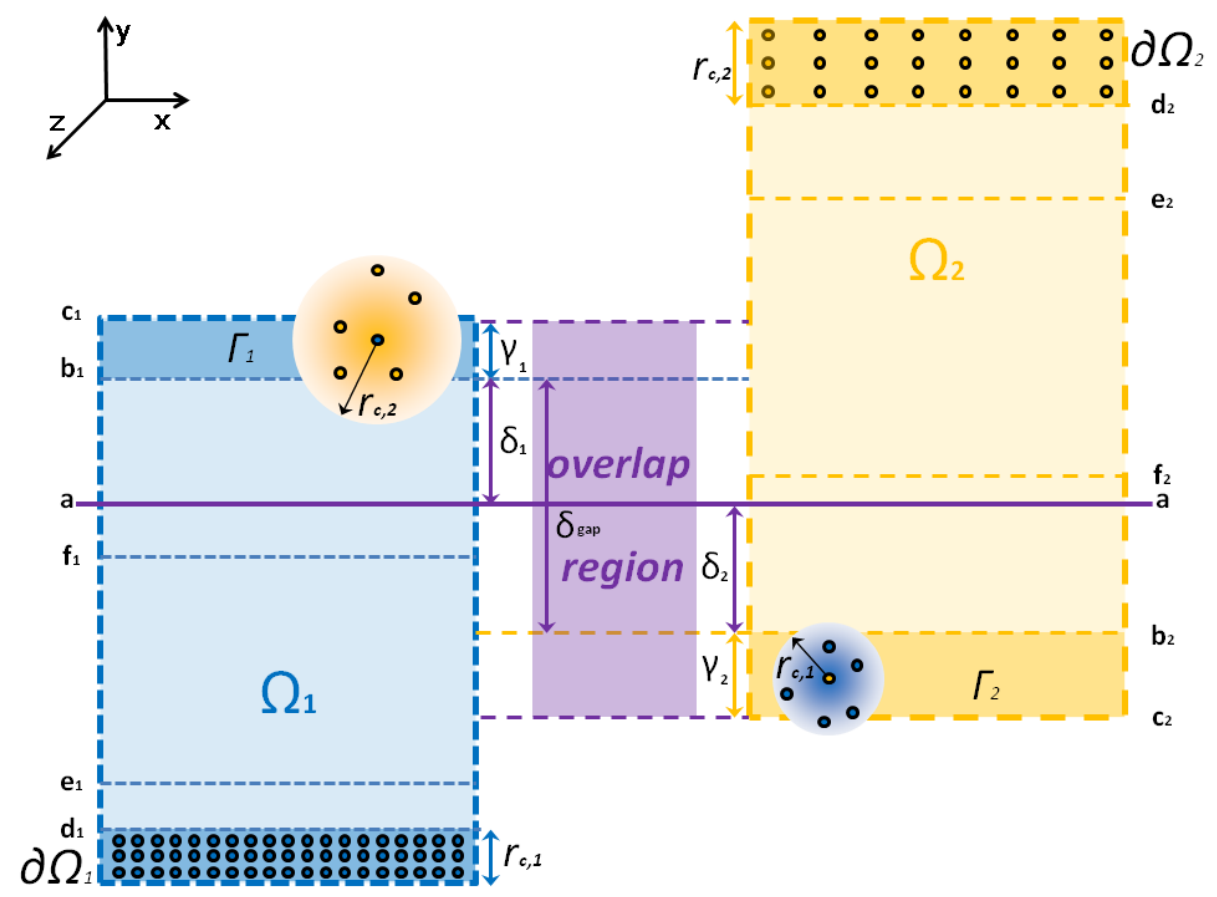

Figure 2: Schematic explanation of the coupled simulation. Sub-domain 1 consists of $\Omega_{1}, \partial \Omega_{1}$ and $\Gamma_{1}$ and sub-domain 2 consists of $\Omega_{2}, \partial \Omega_{2}$ and $\Gamma_{2}$. The two sub-domains are bounded by external boundary conditions (solid wall or periodic) and are coupled by an overlap region. Horizontal lines $a, b_{i}, c_{i}, d_{i}, e_{i}$ and $f_{i}$ are boundaries for different regions. In particular, for $\Omega_{1}$ the artificial interface boundary region $\Gamma_{1}$ extends $\gamma_{1}$ vertically from $b_{1}$ to $c_{1}$. At the bottom below $d_{1}$ we have either periodic or solid wall $\partial \Omega_{1}$ with thickness of $r_{c, 1}$ (represented by frozen particles). Similarly for $\Omega_{2}$ the artificial interface boundary region $\Gamma_{2}$ extends $\gamma_{2}$ vertically from $b_{2}$ to $c_{2}$. At the top above $d_{2}$ we have either periodic or solid wall $\partial \Omega_{2}$ with thickness of $r_{c, 2}$ (represented by frozen particles). In addition, we designate bath regions between $e_{1}$ and $f_{1}$ in $\Omega_{1}$ and between $f_{2}$ and $e_{2}$ in $\Omega_{2}$ to impose constrains for $\Gamma_{2}$ and $\Gamma_{1}$, respectively. A gap of $\delta_{\text {gap }}$ is left between $\Gamma_{1}$ and $\Gamma_{2}$, and it is arbitrarily split into two equal parts with $\delta_{1}=\delta_{2}$. The horizontal line $a$ in the middle of the gap region is referred to as hybrid reference line. Note that $\Omega_{1}$ does not include $\partial \Omega_{1}$ or $\Gamma_{1}$ and $\Omega_{2}$ does not include $\partial \Omega_{2}$ or $\Gamma_{2}$ 
In the context of multi-resolution SPH simulation via domain decomposition, we also consider Dirichlet-type boundary conditions in the artificial boundaries, as shown in Fig. 2. In particular, we constrain the state variables in $\Gamma_{1}$ and $\Gamma_{2}$ of two SPH simulations. For the case of an isothermal fluid, the constraints are performed on density and momentum within $\Gamma_{1}$ and $\Gamma_{2}$. The thickness of external boundary $\partial \Omega_{1}$ is taken as $r_{c, 1}$, which is the cut off radius of SPH simulation performed within sub-domain $1\left(\Omega_{1} \cup \partial \Omega_{1} \cup \Gamma_{1}\right)$. A thinner boundary of $\partial \Omega_{1}$ weakens coupling between fluid and boundary particles while a thicker one beyond cut off does not contribute to the coupling. Similarly, the thickness of external boundary $\partial \Omega_{2}$ is taken as $r_{c, 2}$, which is the cut off radius of SPH simulation performed within sub-domain $2\left(\Omega_{2} \cup \partial \Omega_{2} \cup \Gamma_{2}\right)$. The overlap region is indicated as the shadowed region in Fig. 2, including $\Gamma_{1}, \Gamma_{2}$ and a gap region between them. The gap region is created intentionally so that the constrained values in one artificial boundary are not immediately applied to constrain the other artificial boundary again. Otherwise temporal coupling would be weak (see results of Section 3.1). The length scales in the overlap region should be as follows:

$$
\begin{aligned}
\gamma_{1} & \geq r_{c, 1}, \\
\gamma_{2} & \geq r_{c, 2}, \\
\delta_{\text {gap }} & \geq \max \left(r_{c, 1}, r_{c, 2}\right),
\end{aligned}
$$

where the minimum thickness of artificial boundaries $\Gamma_{1}$ and $\Gamma_{2}$ is taken as the corresponding cut off radius, for the same reason as for the external boundaries. The minimum of the thickness $\delta_{g a p}$ of the gap region is taken as the maximum of the two cut off radii to avoid weak coupling. In practice, we prefer the minimal computation by taking the lower bound values in Eq. (14). Therefore, the thickness of the overlap region is $\delta_{\text {overlap }}=\gamma_{1}+\gamma_{2}+\delta_{\text {gap }}=r_{c, 1}+2 r_{c, 2}$, where $r_{c, 1} \leq r_{c, 2}$. The reasons of choosing such lengths will become more clear as we explain how to constrain state variables based on Lagrangian interpolations in Section 2.4, 2.5 and 2.7.

Since two solutions exist in the overlap region, we arbitrarily split the gap region into two equal parts, that is, $\delta_{1}=\delta_{2}=\delta_{\text {gap }} / 2$, and refer to the middle line $a$ as hybrid reference line. Therefore, the solution of $\Omega_{1}$ below $a$ and the solution of $\Omega_{2}$ above $a$ are glued together to provide the global solution.

Unlike an iterative solver, such as the Jacobi method, the SPH method should rather be understood as a direct solver, so that the final solutions in $\Omega_{1}$ and $\Omega_{2}$ for known external and artificial boundary conditions are obtained without pseudo-time iterations. This makes the domain-decomposition based SPH (DD-SPH) method suitable not only for steady but also for transient problems. In this case, all temporal quantities in Fig. 1 are physical time instead of pseudo-time for iterative relaxation.

\subsection{Dirichlet boundary for momentum in $\Gamma_{1}$ and $\Gamma_{2}$ : constrained particle dynamics}

At $t=t_{\text {comm }}^{n}$, the simulation in sub-domain 1 completes the previous $\left(\Delta t_{c o m m} / \Delta t_{1}\right)$ time steps, where $\Delta t_{1}$ is the time step of SPH simulation in the sub-domain. We impose the velocity constraint within $\Gamma_{1}$ before the simulation proceeds, that is, for every particle $k$ within $\Gamma_{1}$, we calculate its constrained velocity $\mathbf{u}_{k}^{\text {constr }}$ imposed by the simulation in $\Omega_{2}$ by SPH interpolation

$$
\mathbf{u}_{k}^{\text {constr }}=\sum_{l} \frac{\mathbf{v}_{l}}{d_{l}} W_{k l}
$$


where $l$ is the index of particles in $\Omega_{2}$ within a spherical region of radius $r_{c, 2}$ away from particle $k$. Note that $r_{c, 2}$ is selected instead of $r_{c, 1}$, as the former is the characteristic length of dynamics in $\Omega_{2}$. In Eq. (15), $\mathbf{u}_{k}^{\text {constr }}$ can be understood as an interpolated velocity at position $\mathbf{x}_{k}$ in $\Omega_{2}$. This constraint involves particles of $\Omega_{2}$ located in the region between horizontal lines $f_{2}$ to $e_{2}$, as shown in Fig. 2, which is referred to as the bath region of $\Omega_{2}$ for $\Gamma_{1}$. It is simple to see that the thickness of the bath region is $r_{c, 1}+2 r_{c, 2}$. Thereafter, the simulation in $\Omega_{1} \cup \partial \Omega_{1}$ proceeds as usual until $t=t_{c o m m}^{n+1}$.

Similarly, at $t=t_{\text {comm }}^{n}$ the simulation in sub-domain 2 completes the previous $\left(\Delta t_{\text {comm }} / \Delta t_{2}\right)$ time steps, where $\Delta t_{2}$ is the time step of SPH simulation in the subdomain. We impose the velocity constraint within $\Gamma_{2}$ before simulation proceeds, that is, for every particle $k$ within $\Gamma_{2}$, we calculate its constrained velocity $\mathbf{v}_{k}^{\text {constr }}$ imposed by the simulation in $\Omega_{1}$ by SPH interpolation

$$
\mathbf{v}_{k}^{\text {constr }}=\sum_{l} \frac{\mathbf{u}_{l}}{d_{l}} W_{k l}
$$

where $l$ is the index of particles in $\Omega_{1}$ within a spherical region of radius $r_{c, 1}$ away from particle $k$. This constraint involves particles of $\Omega_{1}$ located in the region between horizontal lines $e_{1}$ to $f_{1}$, as shown in Fig. 2, which is referred to as the bath region of $\Omega_{1}$ for $\Gamma_{2}$. It is simple to see that the thickness of the bath region is $r_{c, 2}+2 r_{c, 1}$. Thereafter, the simulation in $\Omega_{2} \cup \partial \Omega_{2}$ proceeds as usual until $t=t_{\text {comm }}^{n+1}$.

From $t_{\text {comm }}^{n}$ to $t_{\text {comm }}^{n+1}$, the velocity $\mathbf{v}_{l}$ in Eq. (15) is a constant copy of values taken from $\Omega_{2}$ at time $t_{\text {comm }}^{n}$. The constraint on $\mathbf{u}_{k}^{\text {constr }}$ is performed at every $\Delta t_{1}$ between $t_{c o m m}^{n}$ and $t_{\text {comm }}^{n+1}$, instead of merely at $t_{\text {comm }}^{n}$ and $t_{\text {comm }}^{n+1}$; likewise, the constraint on $\mathbf{v}_{k}^{\text {constr }}$ in Eq. (16) is performed at every $\Delta t_{2}$ instead of merely at $t_{\text {comm }}^{n}$ and $t_{c o m m}^{n+1}$. This means that the communication between two simulations takes place every $\Delta t_{\text {comm }}$ and the constraints in $\Gamma_{1}$ and $\Gamma_{2}$ are performed at every time step of individual simulations. The frequent constraints in artificial boundaries allow for a rapid convergence of individual simulations in the two sub-domains, which is effective for time-dependent flows, as shown in Section 3.1 and 3.2. For a steady problem, a less frequent constraint may be sufficient.

Although other high-oder Lagrangian interpolations may be utilized in Eqs. (15) and (16), such as the moving least squares method, we adopt SPH interpolation for its simplicity and accuracy level consistent with the SPH simulations. The interpolation kernel in Eqs. (15) and (16) is taken to be the same as the SPH simulation kernel in Eq. (9).

\subsection{Dirichlet boundary for density in $\Gamma_{1}$ and $\Gamma_{2}$ : constrained particle dynamics}

Compressibility is present almost universally in particle-based methods, therefore we further consider density coupling and enable pressure waves across the overlap region.

For any particle $k$ within $\Gamma_{1}$ (likewise for each particle within $\Gamma_{2}$ ), there is no full spherical support for calculating density of particle $k$. There exist a few effective schemes, e.g., in Ref. [18], to correct the density of a particle at or near a boundary. However, these schemes would achieve a local equilibrium density in $\Gamma_{1}$ (and in $\Gamma_{2}$ ), without reflecting any density/pressure variation in the other simulation. 
To couple the dynamics of density/pressure of two simulations, for every particle $k$ within $\Gamma_{1}$ we calculate its constrained density $d_{k}^{\text {cons }}$ imposed by particles in the bath region of $\Omega_{2}$ via SPH interpolation

$$
d_{k}^{\text {cons }}=\sum_{l} \frac{m_{2}}{m_{1}} W_{k l},
$$

where $l$ is the index of particles in $\Omega_{2}$ within a spherical region of radius $r_{c, 2}$ away from particle $k$. Here, $m_{1}$ (constant) is the mass of each particle in sub-domain 1 and $m_{2}$ (constant) is the mass of each particle in sub-domain 2. Similarly, for every particle $k$ within $\Gamma_{2}$ we calculate its constrained density $d_{k}^{\text {cons }}$ imposed by particles in the bath region of $\Omega_{1}$ via SPH interpolation

$$
d_{k}^{\text {cons }}=\sum_{l} \frac{m_{1}}{m_{2}} W_{k l},
$$

where $l$ is the index of particles in $\Omega_{1}$ within a spherical region of radius $r_{c, 1}$ away from particle $k$. The interpolation kernel in Eqs. (17) and (18) is taken to be the same as the SPH simulation kernel in Eq. (9).

Some remarks are in order. The density constraints in the artificial boundaries $\Gamma_{1}$ and $\Gamma_{2}$ indeed allow for propagation of density/pressure variation across the overlap region, as they reflect the dynamic changes of density and hence of the pressure. The density constraint, however, is too restrictive, since the real particle distribution in $\Gamma_{1}$ (similarly in $\Gamma_{2}$ ) does not necessarily correspond to the constrained density. More precisely, the SPH density in $\Gamma_{1}$ based on real particle positions does not necessarily equal to the constrained density. Some kind of relaxation for particle configuration in $\Gamma_{1}$ is definitely needed to resolve this density inconsistency. An ideal relaxation protocol would be: keep particles in $\Omega_{1}$ next to $\Gamma_{1}$ fixed and apply an iterative search for the particle configuration within $\Gamma_{1}$ so that SPH density calculation from Eq. (6) (with a correction for non-spherical support) agrees with the density constraint of Eq. (17) up to a certain tolerance. This iterative procedure, however, would involve the solution of a nonlinear system, size of which is in the order of particle numbers in $\Gamma_{1}$ and its neighborhood. The same iterative procedure would be also needed for $\Gamma_{2}$ and its neighborhood. Even worse, the search for particle configurations in artificial boundaries would be needed at every time of exchanging informations between the two simulations. This procedure would inevitably cause significant computational cost and is not recommended. We have developed an alternative effective procedure, as a result of trying to correct conservative force calculation in $\Gamma_{1}$ and $\Gamma_{2}$, which will be presented in Section 2.6.

Similarly to the consideration of velocity in section 2.4 , the communication between two simulations takes place at every $\Delta t_{\text {comm }}$ and the constraints on density are performed at each time step of individual simulations, that is, every $\Delta t_{1}$ for $\Gamma_{1}$ and $\Delta t_{2}$ for $\Gamma_{2}$.

\subsection{Correction on conservative force in $\Gamma_{1}$ and $\Gamma_{2}$, and particle relaxation}

For every particle $k$ within $\Gamma_{1}$ (likewise for each particle within $\Gamma_{2}$ ), there is no full spherical support for calculating the conservative force $\mathbf{F}_{k}^{C}$ on particle $k$. There exist 
a few methods, e.g., Ref. [18], to correct the calculation of conservative force with non-spherical support. However, these schemes are designed for simulations at steady state and are not proper for coupling transient phenomena.

To allow for dynamic change of pressure in the overlap region, we apply a conditional rule as follows. For every particle $k$ within $\Gamma_{1}$ and its neighboring particle $l$ in sub-domain 1, their normal distances to the exterior boundary (horizontal line $c_{1}$ in Fig. 2) are $h_{k}$ and $h_{l} ; \mathbf{F}_{k l}^{C}$ is their conservative force and $F_{k l}^{C}$ is its magnitude. The tangential components, namely, $\mathbf{F}_{k l}^{C} \cdot \mathbf{e}_{x}$ and $\mathbf{F}_{k l}^{C} \cdot \mathbf{e}_{z}$, remain unchanged between the two particles but the normal components are selected conditionally: if $h_{l} \leq 2 h_{k}, \mathbf{F}_{k l}^{C} \cdot \mathbf{e}_{y}$ is active for particle $k$; otherwise is discarded. The main point of this rule is to satisfy that the sums of pairwise conservative forces on particle $k$ from both sides of $y_{k}$ along $\mathbf{e}_{y}$ direction are similar at equilibrium and present proper variation at non-equilibrium. The same rule applies to particle $l$ : if $h_{k} \leq 2 h_{l}, \mathbf{F}_{l k}^{C} \cdot \mathbf{e}_{y}$ is active for particle l; otherwise is discarded.

The same conditional rule applies to each particle within $\Gamma_{2}$ and its neighboring particles in sub-domain 2 as well.

Due to the conservative formulation adopted in Eq. (10), the pairwise conservative force is repulsive between particles and always promotes regularization of particle configurations [15]. Therefore, as a by-product, the conditional rule also introduces a "natural" relaxation (without any iterative procedure) for the inconsistent particle configurations in $\Gamma_{1}$ and $\Gamma_{2}$, which result from the density over-constraining of Eqs. (17) and (18). As another advantage of the conditional rule, after insertion in the artificial boundary a new particle will not be repelled to exterior immediately (see Section 2.7). The conditional rule also has two drawbacks. Effectively, as a particle $k$ approaches the exterior within $\Gamma_{1}$ (and $\Gamma_{2}$ ), the conditional rule reduces the number of its neighboring particles for calculating the conservative force in the normal direction on particle $k$. Furthermore, the asymmetric operation violates momentum conservation within $\Gamma_{1}$ and $\Gamma_{2}$, similarly to the situation close to a solid boundary. Nevertheless, the conservation within $\Omega_{1}$ and $\Omega_{2}$ are preserved as usual. In this work, we focus on the dynamic feature and computational advantages for particle relaxation of this conditional rule and accept its two drawbacks.

\subsection{Particle deletion and insertion}

In any particle-based approach particles should be able to leave and enter freely the artificial boundaries $\Gamma_{1}$ and $\Gamma_{2}$, which are flow regions but not wall regions. For particle deletion, it is straightforward to simply remove any particle leaving the subdomain. For particle insertion in one sub-domain, since the influxes of momentum and mass are location-dependent, we consider the fluxes at the inserting locations provided by the simulation in the other sub-domain.

\subsubsection{Deletion}

According to the time advancement of simulation in $\Omega_{1} \cup \partial \Omega_{1} \cup \Gamma_{1}$, for every particle $k$ within $\Gamma_{1}$ at time step $t_{1}^{n}$, if $k$ leaves $\Gamma_{1}$ to the exterior during $t_{1}^{n} \longrightarrow t_{1}^{n+1}=t_{1}^{n}+\Delta t_{1}$, that is, it goes outside of line $c_{1}$ in Fig. 2, $k$ is removed at time $t_{1}^{n+1}$. The same deletion applies to the simulation in $\Omega_{2} \cup \partial \Omega_{2} \cup \Gamma_{2}$ but with its own time advancement from $t_{2}^{n} \longrightarrow t_{2}^{n+1}=t_{2}^{n}+\Delta t_{2}$, and a particle is removed at $t_{2}^{n+1}$. 


\subsubsection{Insertion}

For inserting new particles into $\Gamma_{1}$, we select inserting sample points $A_{1}, A_{2}, \ldots, A_{n}$ along the horizontal line $c_{1}$, that is, $y_{A_{i}}=y_{c_{1}}$. For simplicity, we set the sample points with equal distance $\Delta x_{1}$ along $x$ direction, where $\Delta x_{1}$ is the initial particle spacing in $\Omega_{1} \cup \partial \Omega_{1} \cup \Gamma_{1}$. Therefore, we can calculate the density at point $A_{i}$ at time $t_{1}^{n}$ based on data in the bath region of $\Omega_{2}$,

$$
d_{A_{i}}=\sum_{l} \frac{m_{2}}{m_{1}} W_{A_{i}}
$$

where $l$ is the index for $\Omega_{2}$ particles that are within cut off radius $r_{c, 2}$ of position $A_{i}$. Likewise, we are also able to calculate the particle number flux $N_{A_{i}}$ (defined as mass flux divided by $m_{1}$ ) at point $A_{i}$ accumulated from $t_{1}^{0}=0$ to $t_{1}^{n}=n \Delta t_{1}$, based on the momentum flux in $y$ direction at $A_{i}$, which is interpolated from data in the bath region of $\Omega_{2}$ as follows,

$$
\begin{aligned}
\mathbf{u}_{A_{i}} & =\sum_{l} \frac{\mathbf{v}_{l}}{d_{l}} W_{A_{i}}, \\
N_{A_{i}}^{t_{1}^{n}} & =N_{A_{i}}^{t_{1}^{n-1}}+d_{A_{i}} u_{A_{i}, y} \Delta x_{1} \Delta t_{1},
\end{aligned}
$$

where $u_{A_{i}, y}$ is the $y$ component of $\mathbf{u}_{A_{i}}$ and particle number flux $N_{A_{i}}^{t_{1}^{0}}=0$ at time step 0 . Therefore, at $t_{1}^{n}=n \Delta t_{1}$, we insert a new particle at point $A_{i}$, if $N_{A_{i}}^{t_{1}^{n_{1}}} \leq-1 . N_{A_{i}}^{t_{1}^{n}}=N_{A_{i}}^{t_{1}^{n}}+1$ is performed after the insertion. The inserted particle has mass $m_{1}$ and velocity $\mathbf{u}_{A_{i}}$. Note that $u_{A_{i}, y}$ as the $y$ component of the velocity is always downwards so that the inserted particle moves towards $\Omega_{1}$.

The same inserting procedure applies to the simulation in $\Omega_{2} \cup \partial \Omega_{2} \cup \Gamma_{2}$ as well, where the inserting sample points are $B_{1}, B_{2}, \ldots, B_{n}$ along the horizontal line $c_{2}$, that is, $y_{B_{i}}=y_{c_{2}}$. The sample points are with equal distance $\Delta x_{2}$ along $x$ direction, where $\Delta x_{2}$ is the initial particle distance. The only difference from sub-domain 1 is the directional condition.

Therefore, we can calculate the density at point $B_{i}$ at time $t_{2}^{n}$ based on data in the bath region of $\Omega_{1}$.

$$
d_{B_{i}}=\sum_{l} \frac{m_{1}}{m_{2}} W_{B_{i}}
$$

where $l$ is the index for $\Omega_{1}$ particles that are within cut off radius $r_{c, 1}$ of position $B_{i}$. Likewise, we are also able to calculate the particle number flux $N_{B_{i}}$ (defined as mass flux divided by $m_{2}$ ) at point $B_{i}$ accumulated from $t_{2}^{0}=0$ to $t_{2}^{n}=n \Delta t_{2}$, based on the momentum flux in $y$ direction at $B_{i}$, which is interpolated from data in the bath region of $\Omega_{1}$ as follows,

$$
\begin{aligned}
\mathbf{v}_{B_{i}} & =\sum_{l} \frac{\mathbf{u}_{l}}{d_{l}} W_{B_{i}}, \\
N_{B_{i}}^{t_{2}^{n}} & =N_{B_{i}}^{n_{2}^{n-1}}+d_{B_{i}} v_{B_{i}, y} \Delta x_{2} \Delta t_{2},
\end{aligned}
$$

where $v_{B_{i}, y}$ is the $y$ component of $\mathbf{v}_{B_{i}}$ and particle number flux $N_{B_{i}}^{t_{2}^{0}}=0$ at time step 0 . Therefore, at $t_{2}^{n}=n \Delta t_{2}$, we insert a new particle at point $B_{i}$, if $N_{B_{i}}^{t_{2}^{n}} \geq 1$. $N_{B_{i}}^{t_{2}^{n}}=N_{B_{i}}^{t_{2}^{n}}-1$ 
is performed after the insertion. The inserted particle has mass $m_{2}$ and velocity $\mathbf{v}_{B_{i}}$. Note that $v_{B_{i}, y}$ as the $y$ component of the velocity is always upwards so that the inserted particle moves towards $\Omega_{2}$. Recall the asymmetric operation of the conditional rule in Section 2.6, i.e., the newly inserted particles are not repelled to the exterior.

This procedure of particle deletion and insertion applies, in general, to both compressible and incompressible flows. However, for incompressible flows, such as the Wannier flow in Section 3.3, a slightly modified procedure is preferred to have strict mass conservation in each simulation. More precisely, each removed particle is inserted back immediately at the sampling point, which has the maximum inwards particle number flux accumulated. The rest of the operations are the same as in the case for general compressible flows.

We note that the particle deletion and insertion are executed according to different time advancements of the two simulations, that is, they are achieved asynchronously, in contrast to the synchronous deletion and insertion (named as refinement and derefinement) adopted in previous work [11,12]. Therefore, both spatial and temporal speed-ups can be achieved in the sub-domain of low resolution simulation.

A flowchart is depicted in Appendix A to facilitate the understanding of the coupling algorithms.

\section{Results and discussions}

In this section, we perform multi-resolution simulations by the domain decomposition based SPH method (DD-SPH) presented in Section 2. The parameters considered are for liquid water at room temperature with density $\rho_{e q}=10^{3} \mathrm{kgm}^{-3}$, dynamic viscosity $\eta=10^{-3} \mathrm{kgm}^{-1} \mathrm{~s}^{-1}$, and kinematic viscosity $v=10^{-6} \mathrm{~m}^{2} \mathrm{~s}^{-1}$. The artificial sound speed is 10-100 times the characteristic speed of the flow. To test the DD-SPH method, we consider a transient Couette flow, perturbed waves with decaying characteristics, and a two dimensional Wannier flow.

\subsection{Transient Couette flow}

We first consider a transient Couette flow, a simple one-dimensional flow, but useful due to the nonlinear velocity profiles before steady state. We can rely on analytical solutions to verify closely the coupled simulations. A simulation domain is defined with size $L_{x}=L_{z}=2.22 \times 10^{-4} \mathrm{~m}, L_{y}=10^{-3} \mathrm{~m}$ and two parallel walls are perpendicular to $y$ direction. Solid walls in $\partial \Omega_{1}$ and $\partial \Omega_{2}$ are modeled by frozen particles and the no-slip boundary condition is preserved between fluid and solid [14]. Initially fluid particles are at rest and the velocity of bottom wall is set at $v_{w}=9.54 \times 10^{-5} \mathrm{~ms}^{-1}$. The Reynolds number is defined as $R e=v_{w} L_{y} / v=0.0954 ; c_{s}=10 v_{w}$ is taken. Either of the EOS in Eq. (3) can be employed, since we evaluate mainly momentum coupling for this problem.

We start with a trivial case of equal resolutions at two sub-domains in order to establish the baseline of coupled simulations. Thereafter, we consider coupling of two resolutions and quantify the numerical error on temporal coupling. 


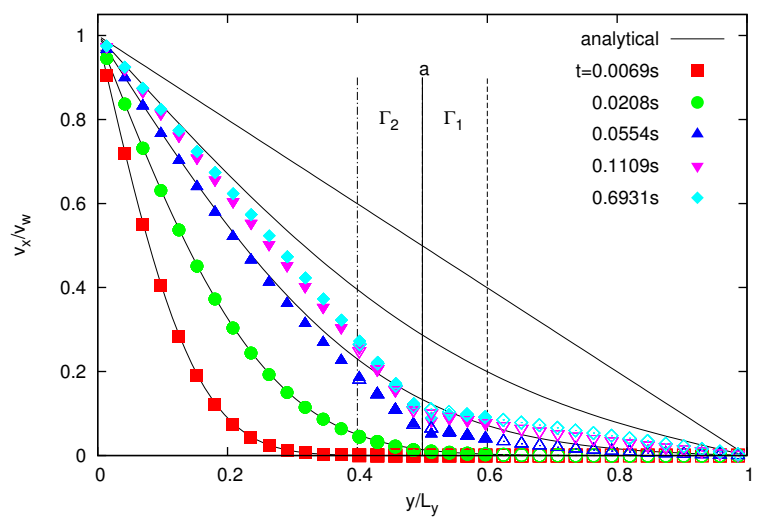

(a) $\delta_{\text {gap }}=0$

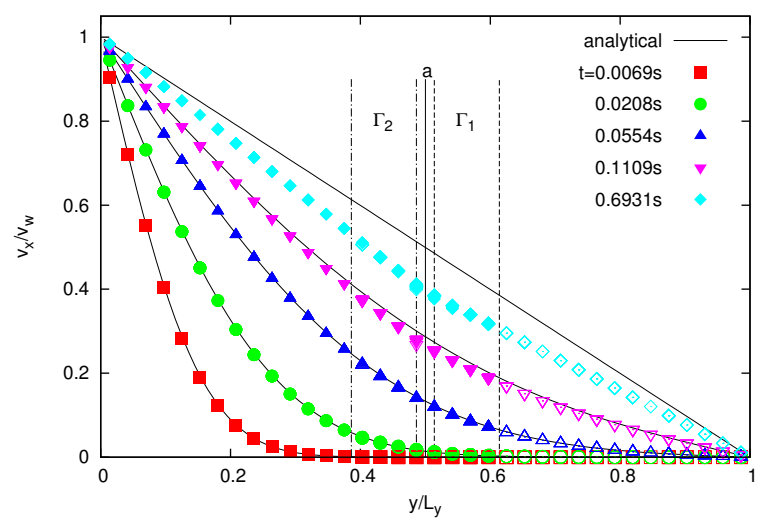

(b) $\delta_{g a p}=r_{c, 1} / 3.6=r_{c, 2} / 3.6$

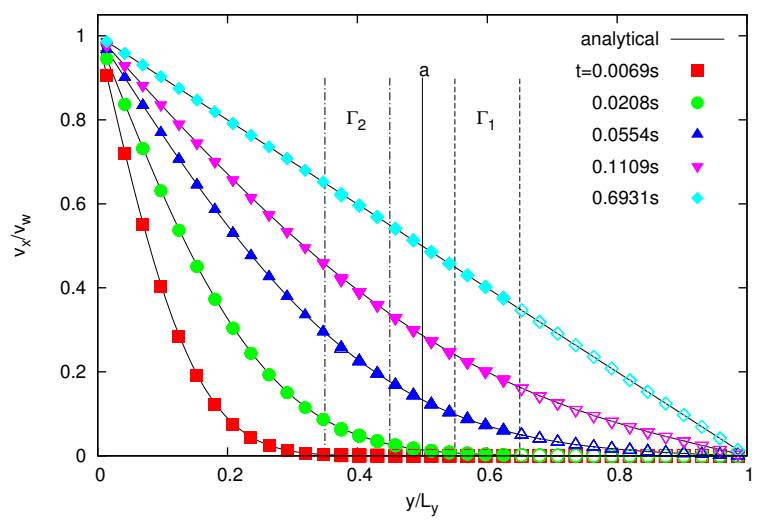

(c) $\delta_{g a p}=\max \left(r_{c, 1}, r_{c, 2}\right)=r_{c, 2}=r_{c, 1}$

Figure 3: Transient Couette flow at two equal resolutions: effect of different $\delta_{\text {gap }}$ on velocity profiles is shown in (a), (b), and (c). $m_{1}=m_{2}, r_{c, 1}=r_{c, 2}, \gamma_{1}=r_{c, 1}, \gamma_{2}=r_{c, 2}, \Delta t_{c o m m}=\Delta t_{1}=\Delta t_{2}$. Solid symbol is for sub-domain 1 on the left and empty symbol is for sub-domain 2 on the right. Definitions of variables are in the text. 


\subsubsection{Equal resolutions}

Initially particles in $\Omega_{1} \cup \partial \Omega_{1} \cup \Gamma_{1}$ are placed on a cubic lattice with $\Delta x_{1}=L_{y} / 36 \approx$ $2.78 \times 10^{-5} \mathrm{~m}$. Similarly, particles in $\Omega_{2} \cup \partial \Omega_{2} \cup \Gamma_{2}$ are also placed on a cubic lattice with $\Delta x_{2}=L_{y} / 36 \approx 2.78 \times 10^{-5} \mathrm{~m}$. Therefore, $m_{1}=\rho_{e q} \Delta x_{1}^{3}=m_{2}=\rho_{e q} \Delta x_{2}^{3}$. The cut off radii are taken as $r_{c, 1}=3.6 \Delta x_{1}, r_{c, 2}=3.6 \Delta x_{2}$ and time steps are $\Delta t_{1}=\Delta t_{2}=1.38622 \times$ $10^{-4} \mathrm{~s}$. Without loss of generality, we take the hybrid reference line $a$ at $y_{a}=L_{y} / 2=$ $5 \times 10^{-4} \mathrm{~m}$ and the overlap region is around $a$ as explained in Fig. 2. Communication for updating boundary conditions in $\Gamma_{1}$ and $\Gamma_{2}$ takes place at each $\Delta t_{\text {comm }}=\Delta t_{2}=\Delta t_{1}$, so that transient behavior is traced closely. We first take sufficiently large artificial boundaries, that is, $\gamma_{1}=r_{c, 1}$ and $\gamma_{2}=r_{c, 2}$. Effects of different gap sizes on the velocity profiles are examined, as shown in Fig. 3. Without sufficient gap size, the coupling of the two sub-domains is not sufficiently strong and the transient results deteriorate with time, as indicated by Fig. 3a and Fig. 3b. In particular, with $\delta_{g a p}=0$, a false steady state is achieved. With $\delta_{g a p}=r_{c, 2} / 3.6=\Delta x_{2}$, the steady state is delayed. The effect of the insufficient gap size can be understood better, if we recall the coupling algorithm in Section 2.4. At $t_{\text {comm }}$, the velocity in $\Gamma_{1}$ is constrained based on velocity in $\Omega_{2} \cup \Gamma_{2}$ at $t_{\text {comm }}$ and the constrained velocity in $\Gamma_{1}$ (or portion of $\Gamma_{1}$ ) is utilized immediately again to constrain the velocity in $\Gamma_{2}$ at $t_{\text {comm }}+\Delta t_{\text {comm }}$. Therefore, the same information is utilized recursively without sufficient relaxation, which results in the weak coupling. In contrast, gap size $\delta_{g a p}=r_{c, 2}=r_{c, 1}$ bypasses using values in $\Gamma_{1}$ or $\Gamma_{2}$ to avoid such ineffective coupling and its result agrees well with the analytical solution [14], as shown in Fig. 3c. The gap condition $\delta_{g a p}=\max \left(r_{c, 1}, r_{c, 2}\right)$ proposed in Section 2.3 proves to be a useful guide to closely couple the transient dynamics of two simulations.

Furthermore, we emphasize that the thickness of artificial boundary should also be sufficient so that information can be effectively propagated towards the interior regions. The effect of different sizes of the artificial boundary is shown in Fig. 4. For smaller artificial boundaries, that is, $\gamma_{1}<r_{c, 1}$ and $\gamma_{2}<r_{c, 2}$, the dynamics of coupled simulation is not captured closely, as shown in Figs. $4 \mathrm{a}$ and $4 \mathrm{~b}$. If the thickness of the artificial boundary is taken as the cut off radius of the sub-domain, the result agrees well with the analytical solution, as shown in Fig. 4c. This is not surprising, as the characteristic length of an artificial boundary should be taken sufficiently large (above the cut off radius), resembling the case of a moving solid boundary [19].

We note that as long as $\delta_{\text {gap }}>0, \gamma_{1}>0$ and $\gamma_{2}>0$, the steady state solution of coupled simulations is not affected. The minimum length scales suggested in the overlap region, however, are crucial to obtain accurately the transient solutions of coupled simulations. After examining the length scales of the overlap region for coupling two equal resolutions, next we focus on coupling of different resolutions.

\subsubsection{Different resolutions}

Based on the setup of section 3.1.1, we increase the resolution in $\Omega_{1} \cup \partial \Omega_{1} \cup \Gamma_{1}$ by taking $\Delta x_{1}=L_{y} / 72$ while the resolution in $\Omega_{2} \cup \partial \Omega_{2} \cup \Gamma_{2}$ remains unchanged. Therefore, $r_{c, 1}=r_{c, 2} / 2, \Delta t_{1}=\Delta t_{2} / 4$ and $m_{1}=m_{2} / 8$. Communication for updating boundary conditions in $\Gamma_{1}$ and $\Gamma_{2}$ takes place at each $\Delta t_{\text {comm }}=\Delta t_{2}$, so that the transient behavior is captured closely. Following the established minimum length criterion in the overlap region, we take $\delta_{g a p}=r_{c, 2}, \gamma_{1}=r_{c, 1}$ and $\gamma_{2}=r_{c, 2}$. Two coupled simulations with hybrid reference line $a$ at either $y_{a}=L_{y} / 2=5 \times 10^{-4} \mathrm{~m}$ or $y_{a}=L_{y} / 4=2.5 \times 10^{-4} \mathrm{~m}$ 


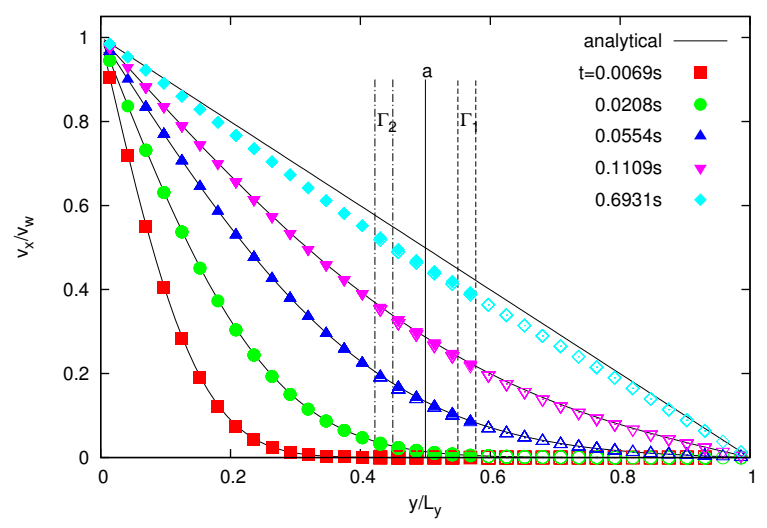

(a) $\gamma_{1}=r_{c, 1} / 3.6$ and $\gamma_{2}=r_{c, 2} / 3.6$

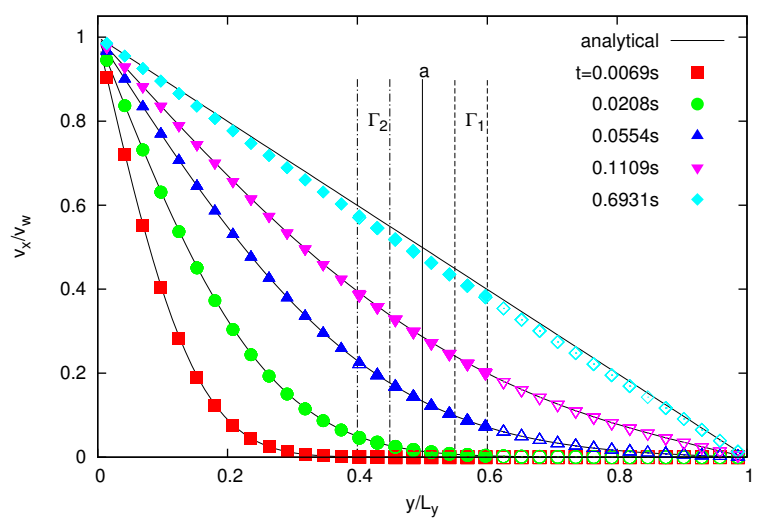

(b) $\gamma_{1}=r_{c, 1} / 2$ and $\gamma_{2}=r_{c, 2} / 2$

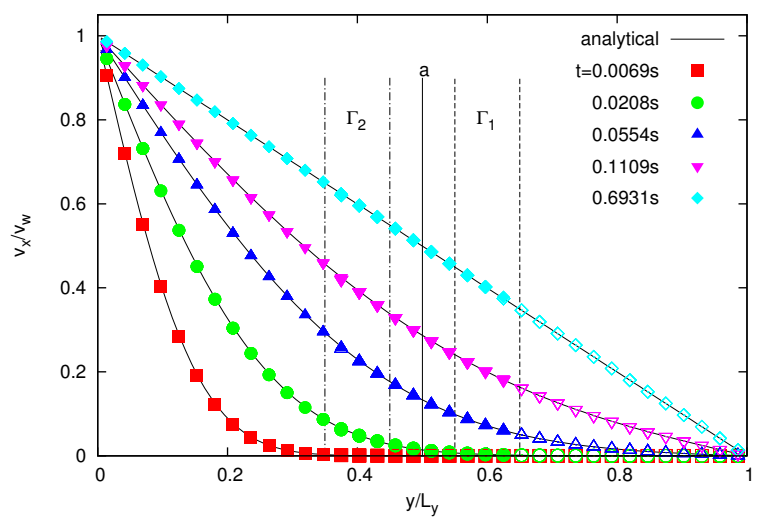

(c) $\gamma_{1}=r_{c, 1}$ and $\gamma_{2}=r_{c, 2}$

Figure 4: Transient Couette flow at two equal resolutions: effect of artificial interface region on velocity profiles is shown in (a), (b), and (c). $m_{1}=m_{2}, r_{c, 1}=r_{c, 2}, \delta_{g a p}=r_{c, 2}, \Delta t_{c o m m}=\Delta t_{1}=\Delta t_{2}$. Solid symbol is for sub-domain 1 on the left and empty symbol is for sub-domain 2 on the right. Definition of variables are in the text. Fig. $4 \mathrm{c}$ is identical to Fig. 3c. 
are performed. Results of both simulations agree well with the analytical solution, as shown in Fig. 5. We observe that simulation results are not sensitive to the location of the overlap region, which may enclose different slopes of velocity profiles.

Compared to the coupled simulation of two equal resolutions in Section 3.1.1, $\Gamma_{1}$ shrinks due to the decreasing cut off radius in sub-domain 1 on the left in Fig. 5. Therefore, the total volume of the overlap region is also reduced. The case with $y_{a}=$ $L_{y} / 4$ is particularly interesting, since the high resolution sub-domain is much smaller than that of the case with $y_{a}=L_{y} / 2$. Minimizing the high resolution cost is beneficial to resolve complex flow with a small amount of computation (see Section 3.3). Therefore, we select the case of $y_{a}=L_{y} / 4$ and investigate in detail the temporal coupling behavior.

We take a snapshot at $t=400 \Delta t_{2}=1600 \Delta t_{1}=0.0554 \mathrm{~s}$ as an example and demonstrate how $\Delta t_{\text {comm }}$ affects the results qualitatively. It is not surprising that increasing $\Delta t_{\text {comm }}$ deteriorates the coupled dynamics as shown in Fig. 6, where results of $\Delta t_{\text {comm }} \geq 64 \Delta t_{2}$ are visually not acceptable at all. To evaluate quantitatively whether results at different $\Delta t_{\text {comm }}$ are good enough, we select three snapshots at early, middle and late development of the flow profile and calculate the relative error expressed in the $L_{2}$ norm. More precisely, we first calculate the velocity differences between SPH particles and analytical solutions at the same locations. Thereafter, the $L_{2}$ norm of the differences is evaluated and normalized by the $L_{2}$ norm of the analytical solutions. The results are shown for $t=0.0208 s, 0.0554 \mathrm{~s}$ and $0.1109 \mathrm{~s}$ in Fig. 7, where the error in general increases as $\Delta t_{\text {comm }}$ increases. We note that the error calculated is the total error including SPH spatial discretization error, time integration error, and the coupling error due to domain-decomposition and its associated constraining interpolations. However, the first two errors depend only on SPH parameters and are fixed values. Therefore, the trend in Fig. 7 reflects the effect of the coupling error. It can also be observed that the error in $\Omega_{1}$ with high resolution (solid symbols) is generally smaller than the error in $\Omega_{2}$ with low resolution (empty symbols). For $t=0.0208 \mathrm{~s}\left(t=150 \Delta t_{2}\right)$, there is no data at $\Delta t_{\text {comm }}=256 \Delta t_{2}$, since $t<\Delta t_{\text {comm }}, \Omega_{1}$ and $\Omega_{2}$ are not coupled for dynamics yet and the error is undefined. Results in Fig. 7 also imply: if one accepts error of $0.6 \%$ for $\Omega_{1}$ and $5 \%$ for $\Omega_{2}$, one may use any value of $\Delta t_{\text {comm }} \leq 8 \Delta t_{2}$ safely, if and only if the error is not accumulated as the coupled simulation proceeds. In fact, the error is a decreasing function of time, as shown in Fig. 8. The coupled simulations show noticeable but acceptable errors for $\Delta t_{\text {comm }} \leq 8 \Delta t_{2}$. For any $\Delta t_{\text {comm }}$, the error diminishes as it approaches steady state, as flow physics tends to be steady at sufficiently long time and any discrepancy between $\Omega_{1}$ and $\Omega_{2}$ is reduced as time proceeds. Finally, for any $\Delta t_{\text {comm }}$, an identical and correct steady solution of the coupled simulations is achieved at sufficiently long time. It is only that larger $\Delta t_{\text {comm }}$ delays the convergence to steady state. If one knows how to measure the error for a particular transient problem, one may choose $\Delta t_{\text {comm }}$ adaptively to keep the error below a certain threshold efficiently.

We note that the errors in Fig. 8 do not decrease strictly monotonically, as the solution approaches to the steady state. There is always a minimal "spike" of the error for each coupled simulation before steady state. However, this spike has nothing to do with the coupling algorithm and is also present for the single resolution SPH simulations, as shown by lines in Fig. 8. The spike is tightly associated with the particular SPH formulation adopted, which carries an inherent mechanism for regularizing parti- 


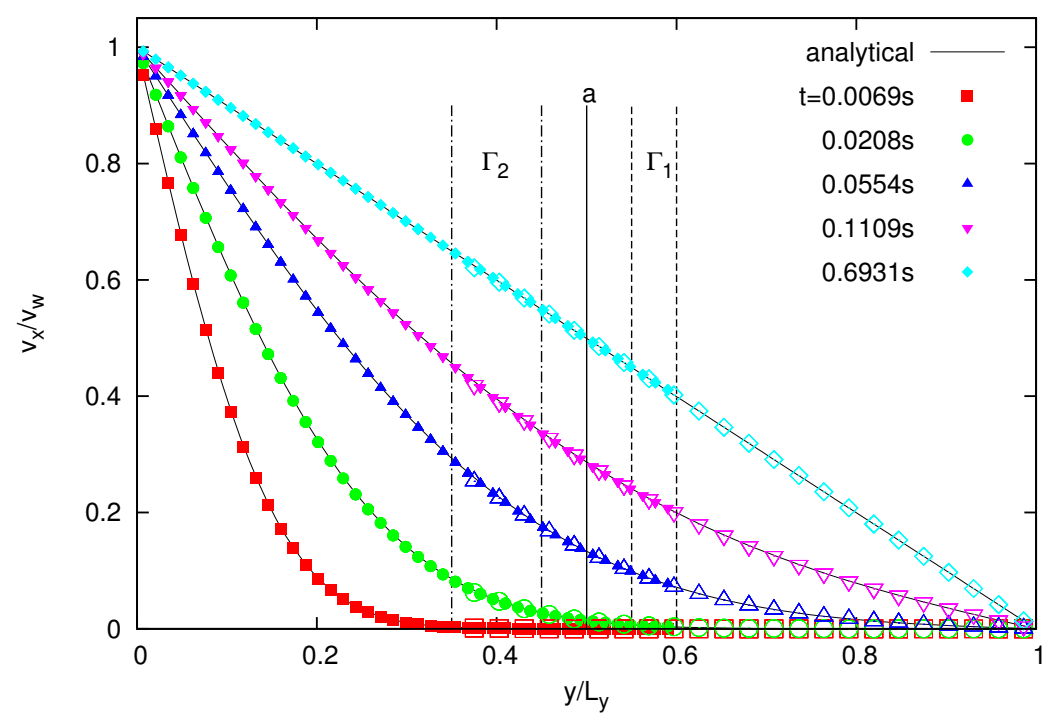

(a) $y_{a}=L_{y} / 2$, overlap region with mild velocity slopes.

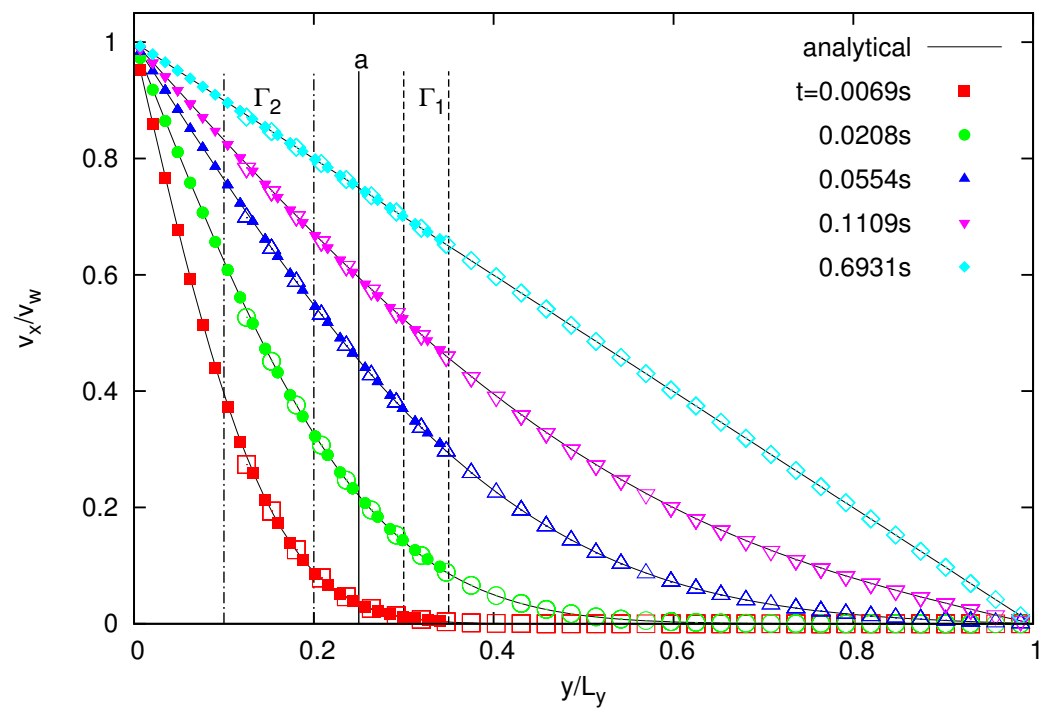

(b) $y_{a}=L_{y} / 4$, overlap region with stiff velocity slopes.

Figure 5: Transient Couette flow at two different resolutions: effect of overlap region at different locations is shown in (a) and (b). $m_{2}=8 m_{1}, r_{c, 2}=2 r_{c, 1}, \gamma_{1}=r_{c, 1}, \gamma_{2}=r_{c, 2}, \delta_{g a p}=r_{c, 2}, \Delta t_{c o m m}=\Delta t_{2}=4 \Delta t_{1}$. Solid symbol is for sub-domain 1 on the left and empty symbol is for sub-domain 2 on the right. 


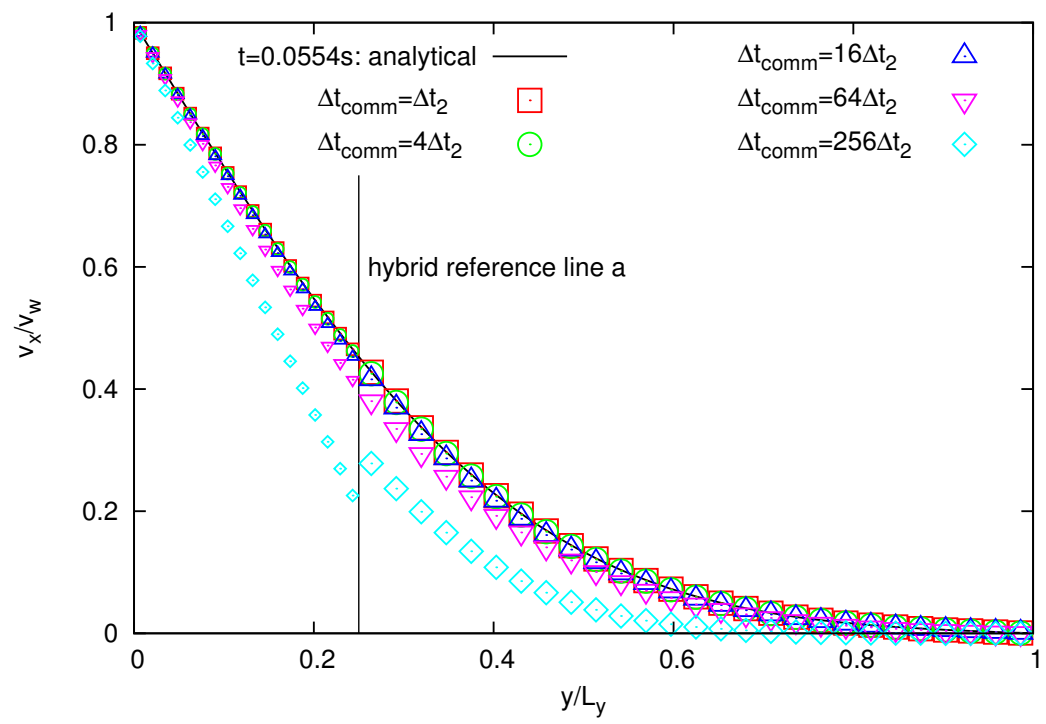

Figure 6: Transient Couette flow at two different resolutions at $t=0.0554 \mathrm{~s}$ : effect of communication time step. $m_{2}=8 m_{1}$ and $\Delta t_{2}=4 \Delta t_{1}$. Small symbol is for $\Omega_{1}$ and large symbol is for $\Omega_{2}$. Values of artificial boundary particles are omitted for clarity.

cle configurations and therefore discretization errors [15]. DD-SPH simulations only shift the time instant of the minimal spike of error. Other SPH formulations (e.g., Ref. [8]) may be adopted to avoid the error spike.

The original domain decomposition method is based on the classical Schwartz alternating relaxation of an iterative solver and it was developed for a steady state problem. Solutions of the two sub-domains become consistent to each other after a sufficiently long pseudo-time of alternating procedure. We adopt a similar idea on a transient flow problem without any pseudo-time relaxation, since the SPH method should be understood as a direct solver in this context. At every $t_{\text {comm }}$ of the coupled simulation, we assume that $\Omega_{1}$ is at quasi-steady state. With known external and artificial boundary conditions, $\Omega_{1}$ is solved by the SPH method with one real time step $\Delta t_{1}$. The same arguments apply to $\Omega_{2}$, which is solved with one real time step $\Delta t_{2}$. The assumption of quasi-steady state at each time instant of the coupling enables us to simulate transient problems.

During the communication time interval $\left[t_{\text {comm }}, t_{\text {comm }}+\Delta t_{\text {comm }}\right)$, the constraints on $\Gamma_{1}$ and $\Gamma_{2}$ are constant values taken at $t_{\text {comm }}$, therefore they always lag behind the transient dynamics for a few time steps. It is possible to linearly extrapolate the time-dependent constrains during the $\Delta t_{\text {comm }}$ for this simple Couette flow. However, this extrapolation would not apply to a more general flow problem, e.g., an oscillatory shear flow. Therefore, we prefer the constant constrains during $\Delta t_{\text {comm }}$ and select $\Delta t_{\text {comm }}=\Delta t_{2}$ for the minimal error of latency.

So far we have evaluated only momentum coupling for a non-equilibrium, accelerating flow problem. In next section, we consider another two non-equilibrium decaying 


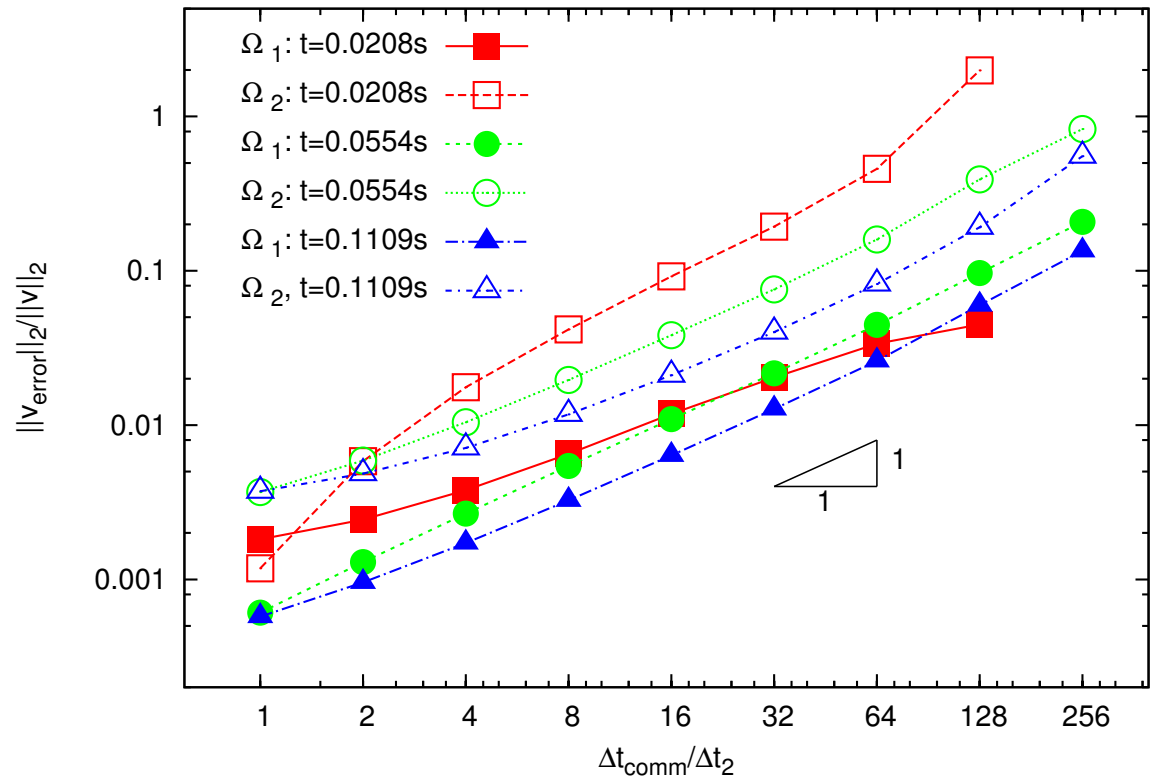

Figure 7: Transient Couette flow at two different resolutions: effect of different communication time steps $\Delta t_{\text {comm }}$ on the relative error at three simulation time instants, corresponding to the early, middle and late development of the velocity profiles. The time steps of two simulations are related as $\Delta t_{2}=4 \Delta t_{1}$. Solid symbol is for $\Omega_{1}$ and empty symbol is for $\Omega_{2}$. The linear slope is guide for the eye. 


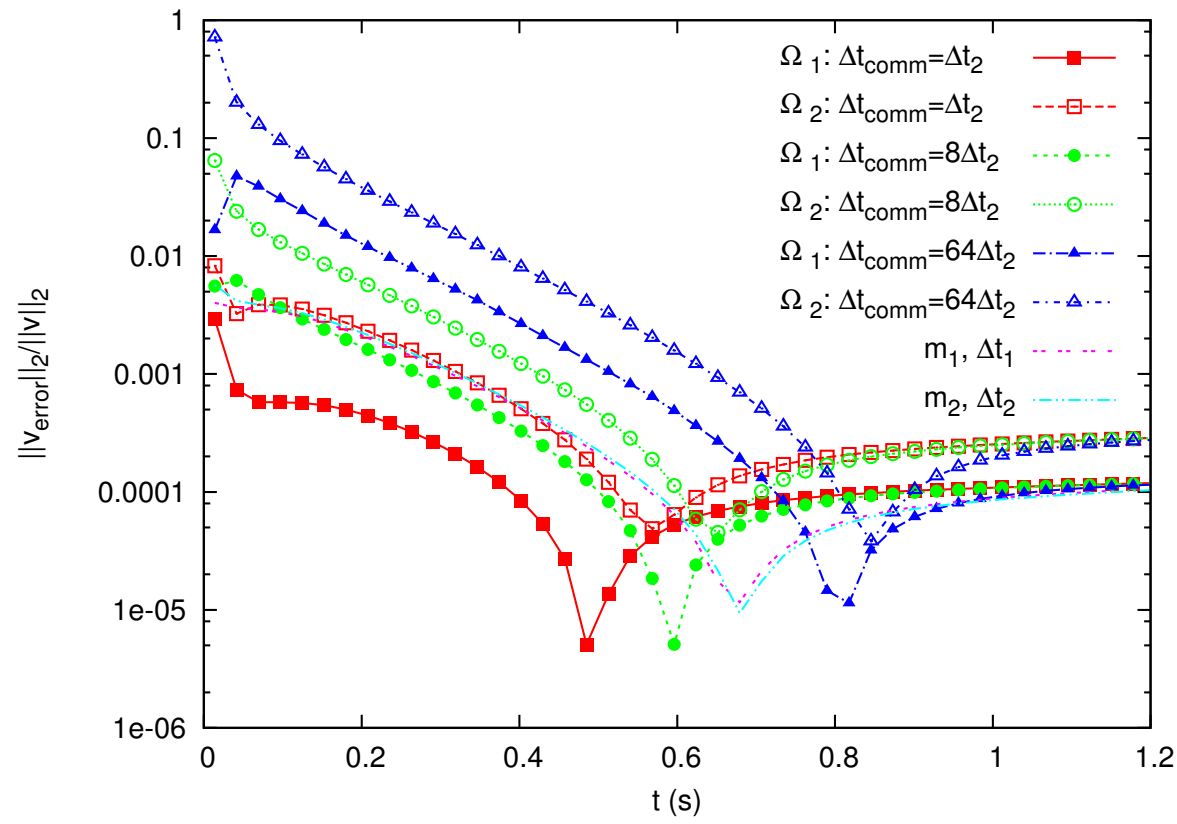

Figure 8: Transient Couette flow at two different resolutions: effect of three communication time steps on the relative error at different simulation time instants. Solid symbol is for $\Omega_{1}$ and empty symbol is for $\Omega_{2}$. In addition, results of two single resolution SPH simulations are also shown by lines, where $\left(m_{1}, \Delta t_{1}\right)$ corresponds to high resolution over the whole domain and $\left(m_{2}, \Delta t_{2}\right)$ corresponds to low resolution over the whole domain. 
flow problems, where the correctness of the coupled simulation does not depend on external walls but solely on the coupling algorithm. In the second decaying problem, we also verify density/pressure coupling against the analytical solution.

\subsection{Perturbation wave of a bulk fluid}

An initial perturbation wave proves to be a useful means to verify numerical schemes $[17,20]$, since the transient solutions at low Reynolds number and low Mach number can be obtained by solving the linearized hydrodynamic equations in Fourier space. This type of non-equilibrium problem is different from the previous Couette flow in the sense that it has a decaying characteristic without new kinetic energy being injected into the system, therefore the simulation result depends solely on the coupling algorithm employed in the overlap region.

Since the external boundary conditions are periodic, we need to couple two subdomains twice in one periodic direction, as illustrated in Fig. 9. Two hybrid interface reference lines are taken at $y_{a}=2 L_{y} / 3$ and $y_{a^{\prime}}=L_{y} / 3$. The wave vector $\mathbf{k}$ is taken along the $y$-direction, which is perpendicular to the hybrid reference lines, namely, $\mathbf{k}=(0, k, 0)$. We consider both transversal and parallel waves, the velocity of which is either perpendicular or parallel to the wave vector. The former verifies the momentum coupling while the latter verifies the density coupling and pressure across the overlap region. The simulation domain has size $L_{x}=L_{z}=2.22 \times 10^{-4} \mathrm{~m}$ and $L_{y}=2.0 \times 10^{-3} \mathrm{~m}$. We focus on one periodic wave with $k=2 \pi / L_{y}$, as resolving an even smaller wave length is just a matter of higher resolution.

\subsubsection{Transversal wave}

The fluid domain is perturbed with an initial velocity $v_{x}(t=0)=v_{0} \sin (k y)$. Velocities in the other two directions are unperturbed as $v_{y}(t=0)=v_{z}(t=0)=0$. To maintain validity of the analytical solution, $v_{0}=9.54 \times 10^{-5} \mathrm{~ms}^{-1}$ is selected so that Reynolds number $R e=v_{0} L_{y} / v=0.181$. The sound speed $c_{s}=9.54 \times 10^{-3} \mathrm{~ms}^{-1}$ is taken so that Mach number $M_{a}=v_{0} / c_{s}=0.01$ is also small. The analytical solution is [20]:

$$
\begin{aligned}
& v_{x}(t)=v_{0} \sin (k y) \exp \left(-v k^{2} t\right) \\
& v_{y}(t)=v_{z}(t)=0,
\end{aligned}
$$

where $v_{x}$ is an exponentially decaying function of the product of kinematic viscosity, wave number squared and time. The velocity in the other two directions remains unchanged with time. We start with a trivial DD-SPH simulation, where resolutions of two sub-domains are equal, that is, $\Delta x_{1}=\Delta x_{2}=L_{y} / 72, r_{c, 1}=r_{c, 2}=L_{y} / 20$, and $\Delta t_{1}=\Delta t_{2}=1.38622 \times 10^{-4} \mathrm{~s}$. Communication for updating the artificial boundary conditions in $\Gamma_{1}$ (or $\Gamma_{1}^{\prime}$ ) and $\Gamma_{2}$ (or $\Gamma_{2}^{\prime}$ ) takes place at each $\Delta t_{\text {comm }}=\Delta t_{1}=\Delta t_{2}$, so that the transient behavior is tracked closely. The result of DD-SPH simulation with equal resolutions agrees very well with the time-dependent analytical solution, as shown in Fig. 10a, and is almost identical to a single SPH simulation with the same resolution (results not shown). The result implies that as long as numerical resolution is sufficient to resolve the wave length, the coupling algorithm in DD-SPH method does not compromise any physics. 


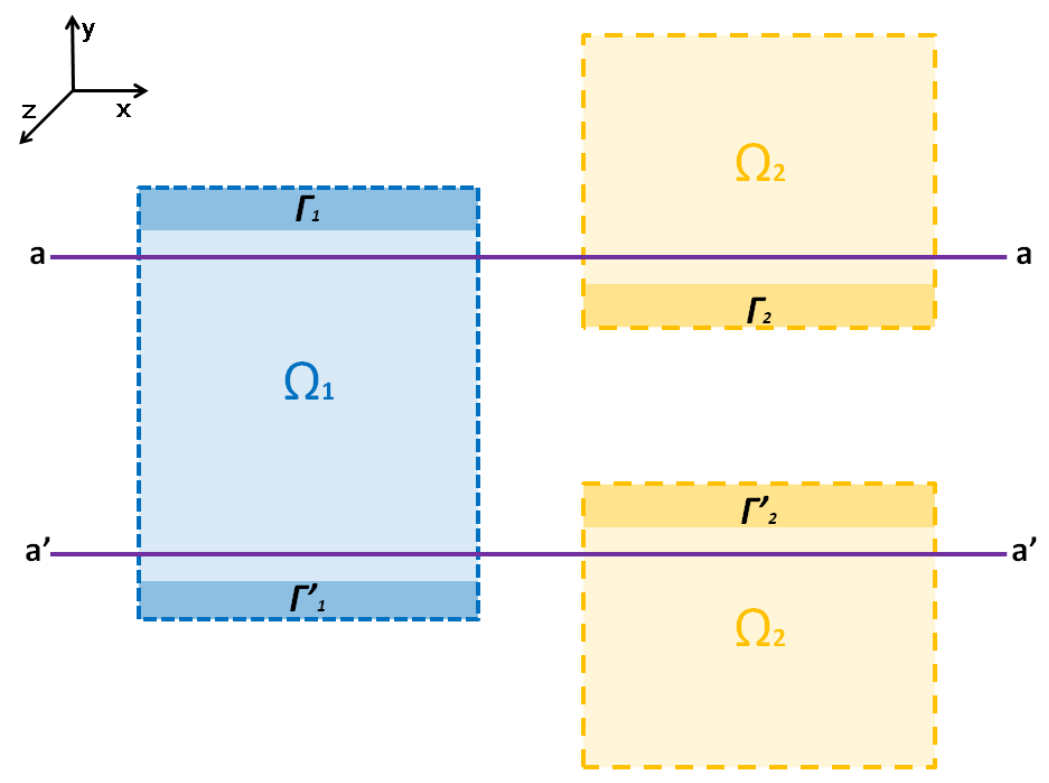

Figure 9: Schematic of coupling in one periodic direction. Periodic boundaries are applied in all directions and the coupling between sub-domains is performed twice in $y$ direction. The first overlap region is around hybrid reference line $a$ and the second overlap region is around hybrid reference line $a^{\prime}$. The associated artificial boundaries are $\Gamma_{1}, \Gamma_{2}$ for $a$ and $\Gamma_{1}^{\prime}, \Gamma_{2}^{\prime}$ for $a^{\prime}$, respectively, as explained in Fig. 2. 


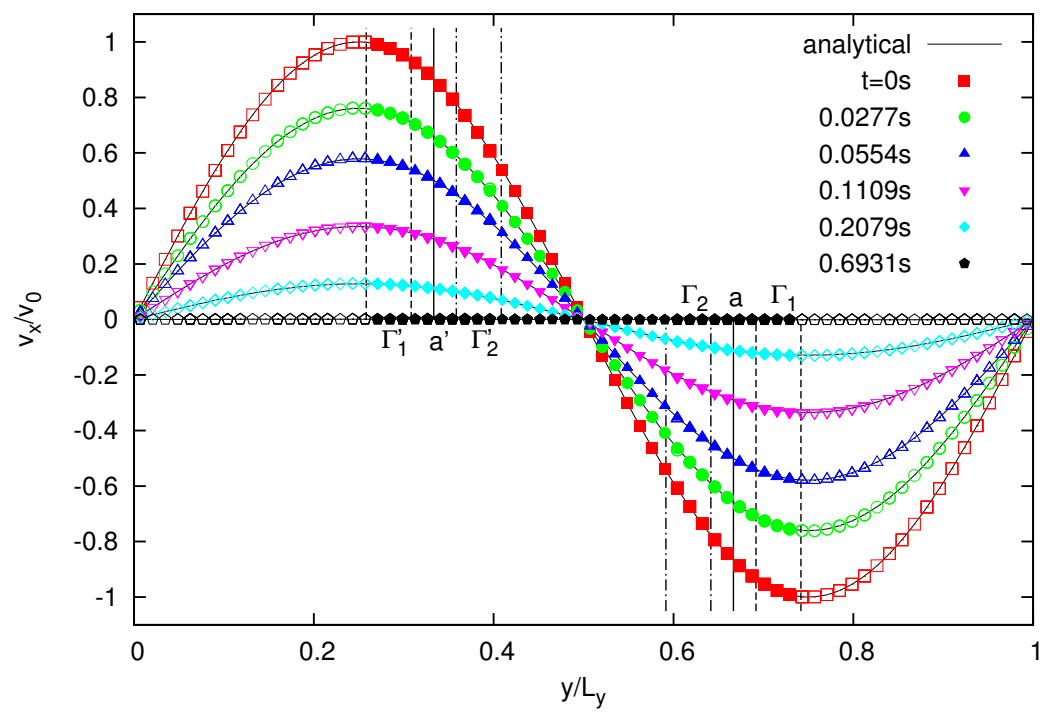

(a) $m_{1}=m_{2}$ and $\Delta t_{1}=\Delta t_{2}$

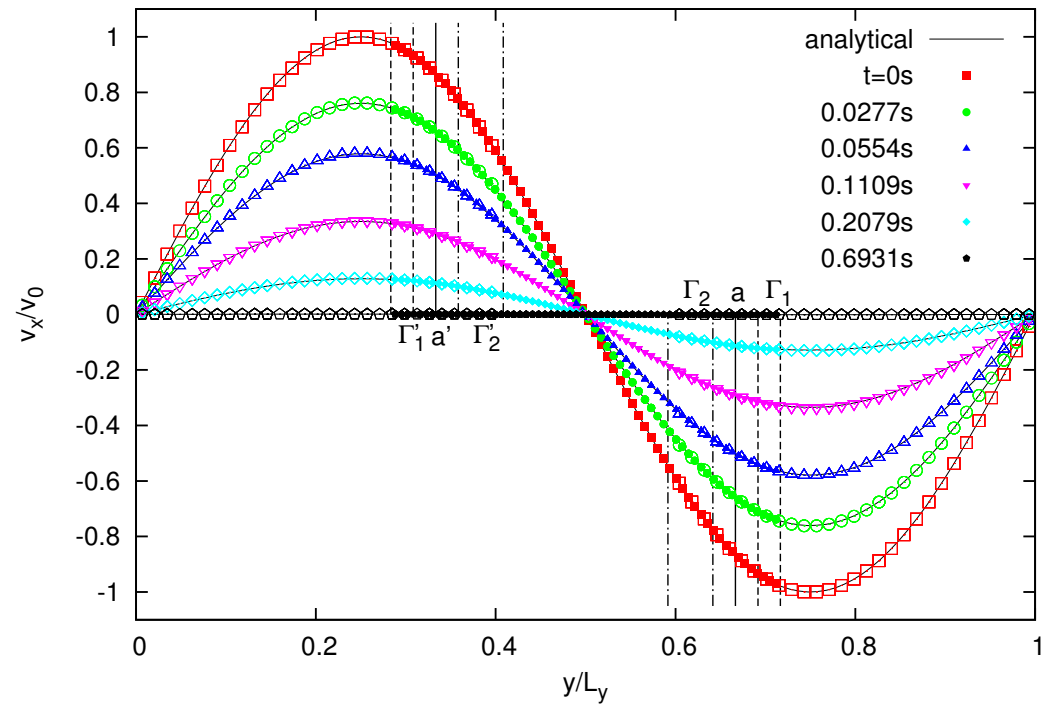

(b) $m_{2} / m_{1}=8$ and $\Delta t_{2} / \Delta t_{1}=4$

Figure 10: Transversal wave: transient velocity profiles for two coupled simulations are shown in (a) and (b). $\rho_{k}^{\text {constr }}=m_{l} \sum_{l} W_{k l}$ and $\left(\mathbf{F}_{k}^{C} \cdot \mathbf{e}_{y}\right)$ conditional. Particle $k \in \Gamma_{1}, \Gamma_{2}, \Gamma_{1}^{\prime}$, or $\Gamma_{2}^{\prime}$. Solid symbol is for the middle sub-domain and empty symbol is for the side sub-domain. 
Furthermore, we increase the resolution in $\Omega_{1} \cup \Gamma_{1} \cup \Gamma_{1}^{\prime}$ by taking $\Delta x_{1}=L_{y} / 144$ while the resolution in $\Omega_{2} \cup \Gamma_{2} \cup \Gamma_{2}^{\prime}$ remains unchanged. Therefore, $m_{1}=m_{2} / 8$, $r_{c, 1}=r_{c, 2} / 2$, and $\Delta t_{1}=\Delta t_{2} / 4$. Communication for updating boundary conditions in $\Gamma_{1}\left(\right.$ or $\left.\Gamma_{1}^{\prime}\right)$ and $\Gamma_{2}$ (or $\Gamma_{2}^{\prime}$ ) takes place at each $\Delta t_{\text {comm }}=\Delta t_{2}$. The result of DD-SPH simulation with different resolutions, as shown in Fig. 10b, still matches very well the analytical solution, which proves that accurate momentum coupling is obtained for this type of non-equilibrium problem with a decaying characteristic.

We note that for the transversal wave, different combinations of density correction or constraint and conservative force correction in $\Gamma_{1}\left(\right.$ or $\left.\Gamma_{1}^{\prime}\right)$ and $\Gamma_{2}\left(\right.$ or $\left.\Gamma_{2}^{\prime}\right)$ do not affect the velocity profiles significantly, since only the momentum coupling in Eqs. (15) and (16) matters. Therefore, we only show results of the constrained density (Section 2.5) together with the corrected conservative force by the conditional rule (Section 2.6) in the artificial boundaries.

\subsubsection{Parallel wave}

We further consider a velocity perturbation applied parallel to the wave vector and perpendicular to the hybrid interface, that is, $v_{y}(t=0)=v_{0} \sin (k y)$ while the other two directions remain unperturbed with $v_{x}(t=0)=v_{z}(t=0)=0$. All other parameters of the parallel wave are identical as in the case of the transversal wave. The analytical solution reads [20]:

$$
\rho(t)=\rho_{e q}\left[1-M_{a} \cos (k y) \sin \left(c_{s} k t\right) \exp \left(-2 v k^{2} t / 3\right)\right] .
$$

The overall magnitude of the oscillation is bounded by Mach number $M_{a}$ and decays exponentially with time. Since the velocity travels along the wave vector ( $y$ direction), initially the fluid is compressed in one half of the domain $\left(L_{y} / 4<y<3 L_{y} / 4\right)$ and is stretched in the other half of the domain $\left(y<L_{y} / 4\right.$ and $\left.y>3 L_{y} / 4\right)$. Compression and elongation of the fluid alternate as velocity evolves with time. Therefore, we can adopt this problem to investigate the dynamic change of density in a DD-SPH simulation. At first, we consider a trivial case, where the resolutions of two sub-domains are equal, that is, $\Delta x_{1}=\Delta x_{2}=L_{y} / 72, r_{c, 1}=r_{c, 2}=L_{y} / 20$, and $\Delta t_{1}=\Delta t_{2}=1.38622 \times 10^{-4} s$. Communication for updating artificial boundary conditions in $\Gamma_{1}\left(\right.$ or $\left.\Gamma_{1}^{\prime}\right)$ and $\Gamma_{2}\left(\right.$ or $\left.\Gamma_{2}^{\prime}\right)$

takes place at each $\Delta t_{\text {comm }}=\Delta t_{1}=\Delta t_{2}$. If the density in the artificial boundaries are set to the equilibrium value, the coupling of density fields in two sub-domains is completely ignored. Therefore, it is not surprising that the density profile deteriorates from the artificial boundary towards the interior of the sub-domain as simulation time proceeds, as shown in Fig. 11. Any correction on the conservative force calculation in the artificial boundary does not help, as the correction is again not based on the dynamics of the other sub-domain. For example, applying either an equilibrium conservative force or a conditional conservative force in the normal direction in the artificial boundaries does not improve the results, as shown in Figs. 11a and 11b.

Alternatively, we apply Eqs. (17) and (18) to constrain the density dynamically in the artificial boundaries of one simulation based on the information of the other simulation. Intuitively, this operation makes sense since the dynamics of density in one simulation is reflected and propagated in the other simulation. Together with the density constraint, we assume that the normal component of the conservative force in the 


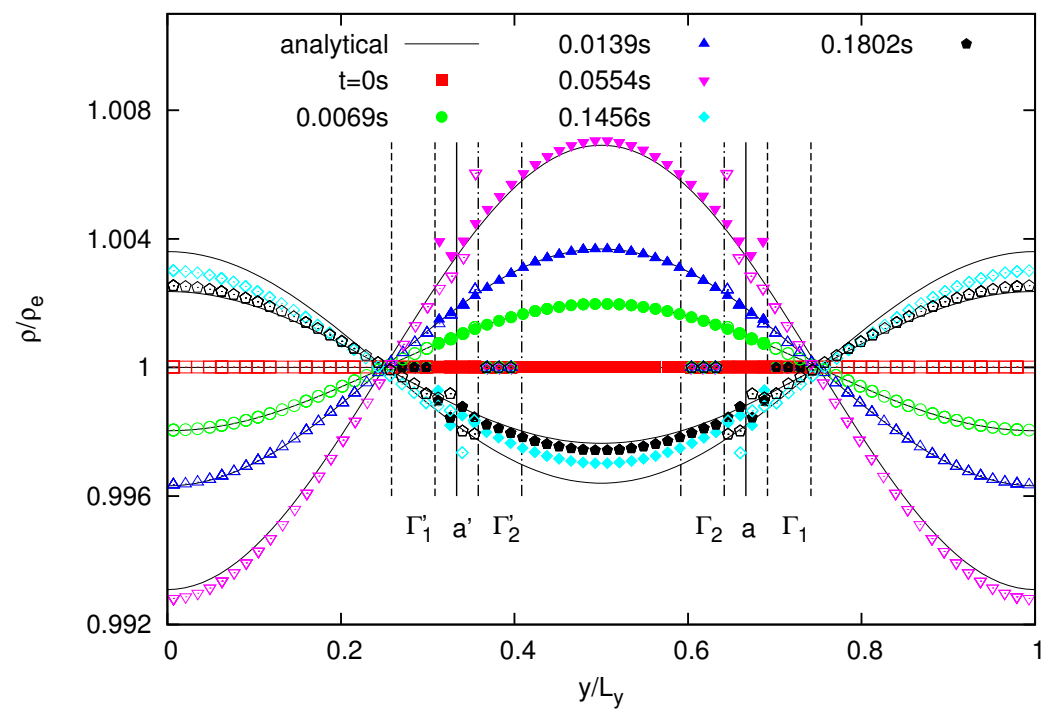

(a) $\rho_{k}=\rho_{e q}$ and $\mathbf{F}_{k}^{C} \cdot \mathbf{e}_{y}=0$

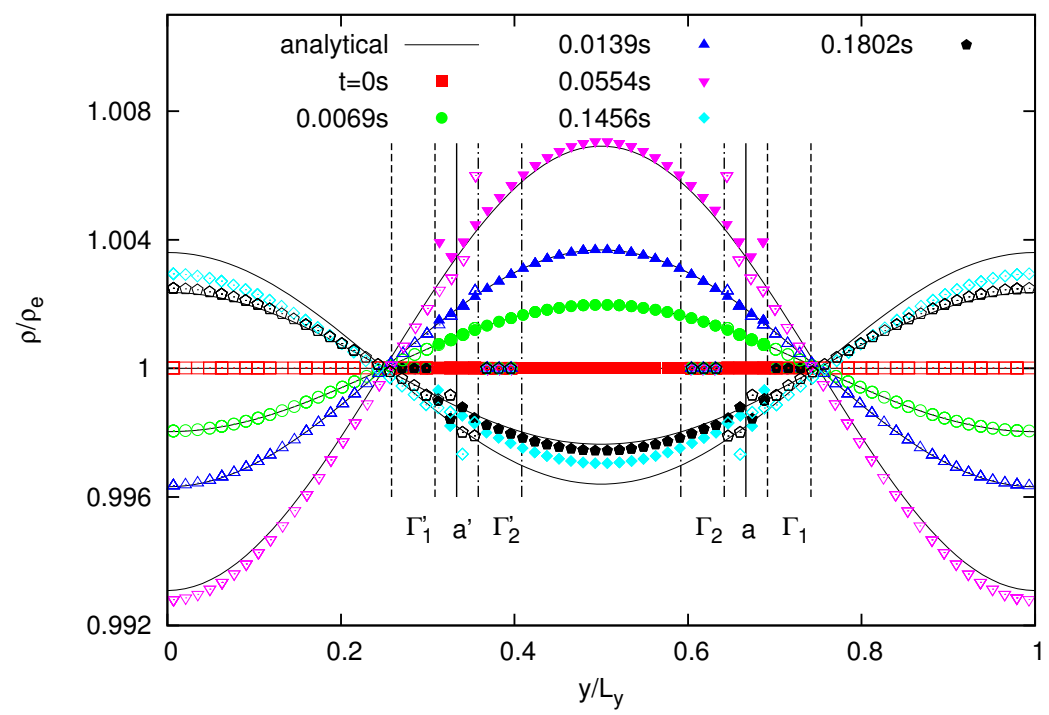

(b) $\rho_{k}=\rho_{e q}$ and $\left(\mathbf{F}_{k}^{C} \cdot \mathbf{e}_{y}\right)$ conditional

Figure 11: Parallel wave: transient density profiles at two equal resolutions with the equilibrium density at the artificial boundaries are shown in (a) and (b). $m_{1}=m_{2}, \Delta t_{1}=\Delta t_{2}$ and EOS with Eq. (3b). Density is set to be the equilibrium value at the artificial boundaries. In (a), the conservative force is set to the equilibrium value in the normal direction. In (b), the conservative force is corrected by the conditional rule in the normal direction. Particle $k \in \Gamma_{1}, \Gamma_{2}, \Gamma_{1}^{\prime}$, or $\Gamma_{2}^{\prime}$. Solid symbol is for the middle sub-domain and empty symbol is for the side sub-domain. 
artificial boundaries vanishes as in the equilibrium. Results shown in Fig. 12a, indicate a significant improvement over the previous choices in Fig. 11. It can be observed that the dynamics of the density is indeed coupled and it propagates gracefully across the overlap region, even after the alternating period between the fluid compression (high density) and elongation (low density). Since the pressure is determined by the EOS, the effective coupling of the density field also means that the pressure wave propagates across the two simulations naturally. There is a slight mismatch, however, as indicated by arrows in Fig. 12a, in the gap region between artificial boundaries. This small inconsistency results from constraining both velocity and density at the same time in the artificial boundaries. More precisely, the velocity constraint on particles in $\Gamma_{1}$ determines their trajectories. The SPH calculation of density in $\Gamma_{1}$ and its neighborhood would yield slightly different density field from the one obtained from the constrained density. In other words, the density constraint is a bit too strong, causing the discrepancy between the constrained value and the actual particle configuration in $\Gamma_{1}$. The same argument applies to particles in $\Gamma_{2}, \Gamma_{1}^{\prime}$, and $\Gamma_{2}^{\prime}$. It is expected that the discrepancy would be amplified in longer time.

As mentioned in Section 2.6, one of the features about the conditional conservative force at the artificial boundary is to regularize the particle configuration according to the density. Hence, the previous minor inconsistency can be completely removed, if we apply the conditional rule to correct the calculation of conservative force in the artificial boundaries, as shown in Fig. 12b.

After establishing the baseline for coupling equal resolutions, we further consider a DD-SPH simulation with different resolutions by increasing the resolution in $\Omega_{1} \cup$ $\Gamma_{1} \cup \Gamma_{1}^{\prime}$ to be $\Delta x_{1}=5$. The resolution in $\Omega_{2} \cup \Gamma_{2} \cup \Gamma_{2}^{\prime}$ remains unchanged. Therefore, $m_{1}=m_{2} / 8, r_{c, 1}=r_{c, 2} / 2$, and $\Delta t_{1}=\Delta t_{2} / 4$. Communication for updating boundary conditions in $\Gamma_{1}$ (or $\Gamma_{1}^{\prime}$ ) and $\Gamma_{2}$ (or $\Gamma_{2}^{\prime}$ ) takes place at each $\Delta t_{\text {comm }}=\Delta t_{2}$. Results are shown in Fig. 13, where density fields are matched accurately between the two simulations with different resolutions. A slightly larger value of density inside $\Gamma_{2}$ and $\Gamma_{2}^{\prime}$ is observed, which is different from the discrepancy resulted from density overconstraining mentioned above. The small deviation of density inside $\Gamma_{2}$ and $\Gamma_{2}^{\prime}$ in Fig. 13 is due to the bias in the SPH interpolation for density on non-random locations. This is a well known artifact of the SPH interpolation and can be corrected empirically [16]. Other higher order Lagrangian interpolations, such as the moving least squares method, may avoid such a bias in the interpolation.

Finally, we note that the linear EOS in Eq. (3a), which has been widely used in simulations of steady state flows, e.g., Ref. [14], is not proper to study the transient behavior of the density dynamics from the parallel wave perturbation, as shown in Fig. 14. The erroneous result is due to its non-stiffness, which causes the pressure not to respond correctly to any dynamic change of the density.

Until now, we have considered only one-dimensional flow problems. Even though in the simulation of the parallel wave perturbation the particle momentum is in the normal direction to the hybrid interface, no particle deletion and insertion operations were actually needed for the short time behavior. In the next section, we will consider a two dimensional flow problem, where mass and momentum fluxes are significant across the hybrid interface, and this serves as a testbed to validate the asynchronous particle deletion and insertion operations. 


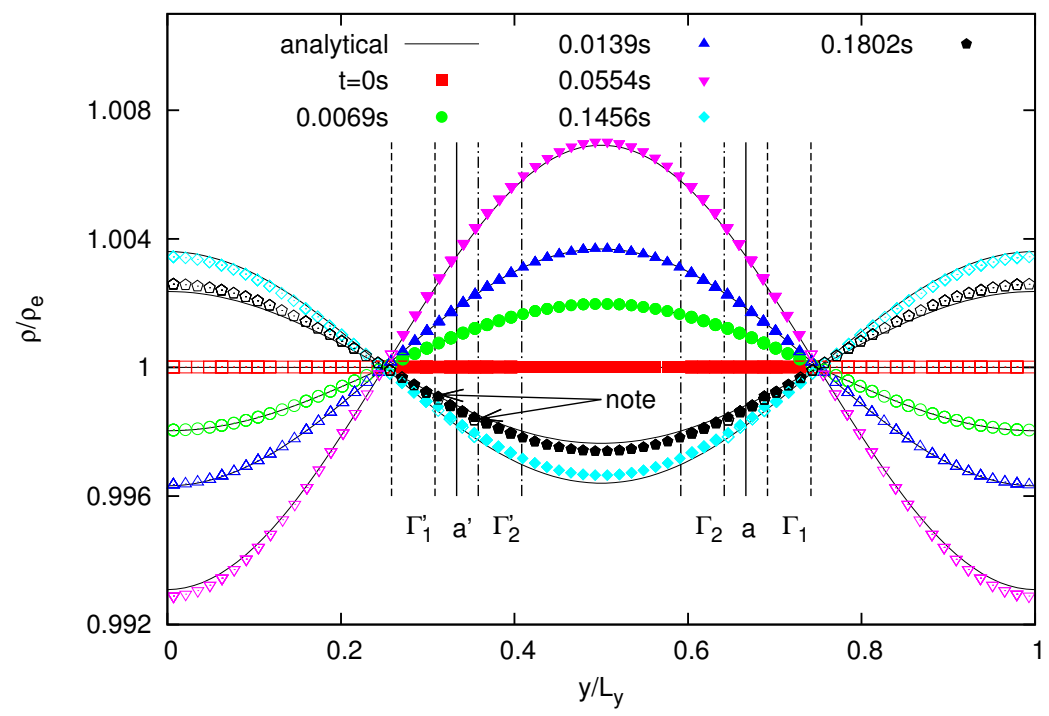

(a) $\rho_{k}^{\text {constr }}=m_{l} \sum_{l} W_{k l}$ and $\mathbf{F}_{k}^{C} \cdot \mathbf{e}_{y}=0$

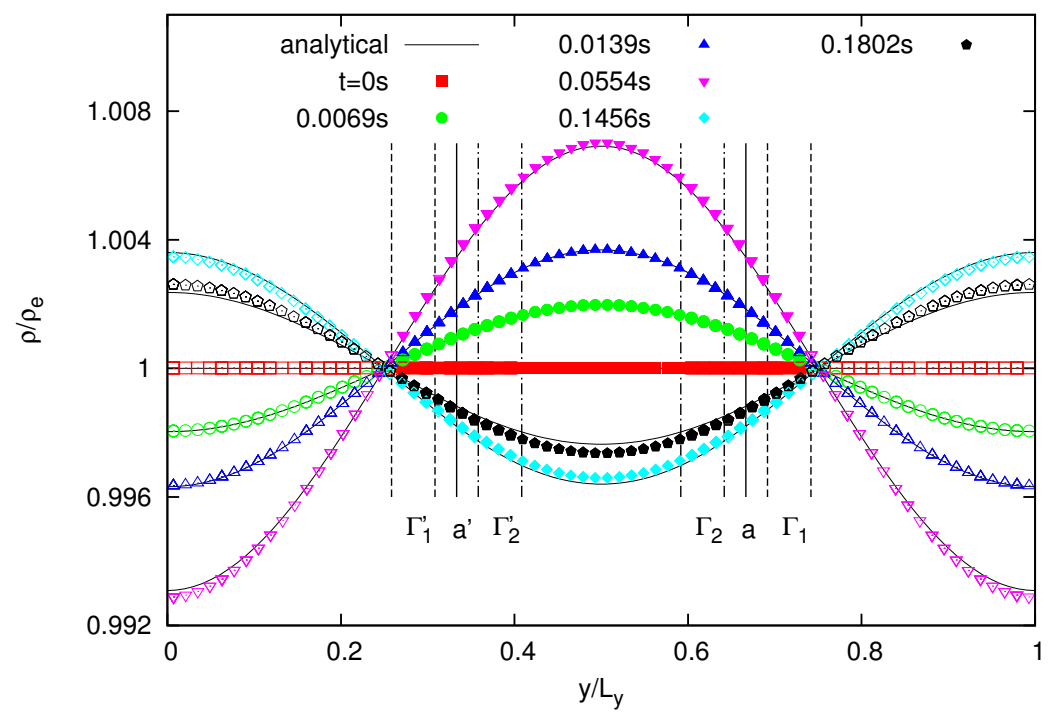

(b) $\rho_{k}^{\text {constr }}=m_{l} \sum_{l} W_{k l}$ and $\left(\mathbf{F}_{k}^{C} \cdot \mathbf{e}_{y}\right)$ conditional

Figure 12: Parallel wave: transient density profiles at two equal resolutions with the constrained density at the artificial boundaries are shown in (a) and (b). $m_{1}=m_{2}, \Delta t_{1}=\Delta t_{2}$ and EOS with Eq. (3b). Density is constrained at artificial boundaries based on information of the other sub-domain. In (a), the conservative force is set to the equilibrium value in the normal direction. In (b), the conservative force is corrected by the conditional rule in the normal direction. Particle $k \in \Gamma_{1}, \Gamma_{2}, \Gamma_{1}^{\prime}$, or $\Gamma_{2}^{\prime}$. Solid symbol is for the middle sub-domain and empty symbol is for the side sub-domain. 


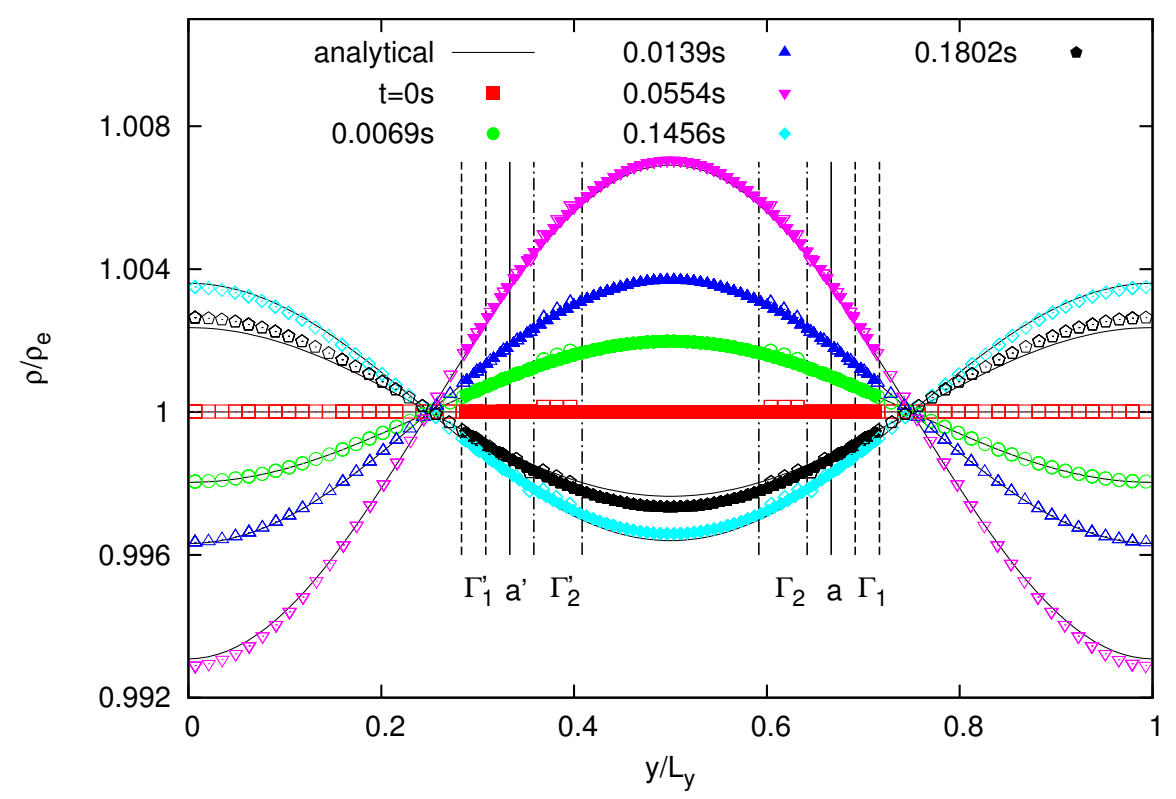

Figure 13: Parallel wave: transient density profiles at two resolutions with the constrained density and the conditional conservative force applied in the artificial boundaries. $m_{2} / m_{1}=8 ; \Delta t_{2} / \Delta t_{1}=4$. EOS with Eq. (3b). Solid symbol is for the middle sub-domain and empty symbol is for the side sub-domain. 


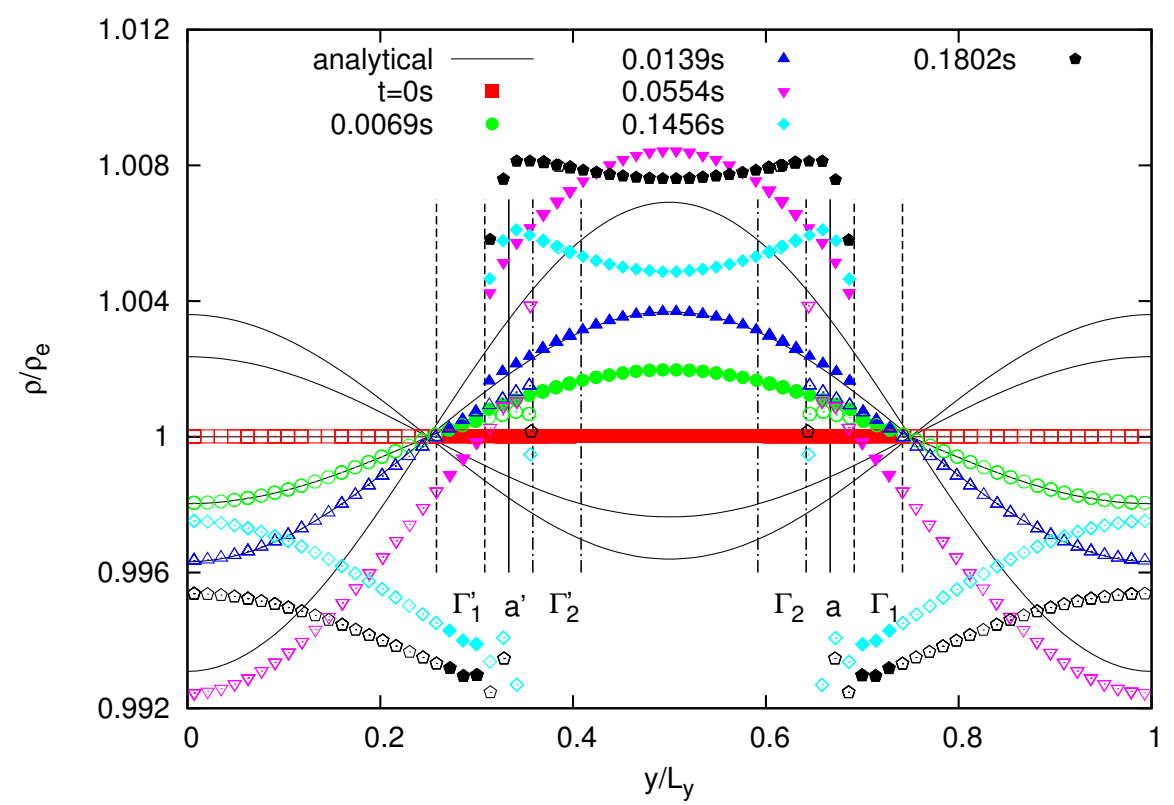

Figure 14: Parallel wave: effect of equation of state (EOS) on the transient density profiles. The linear EOS in Eq. (3a) is applied. The constrained density and the conditional conservative force are applied at the artificial boundaries. $m_{1}=m_{2}, \Delta t_{1}=\Delta t_{2}$. Solid symbol is for the middle sub-domain and empty symbol is for the side sub-domain. 


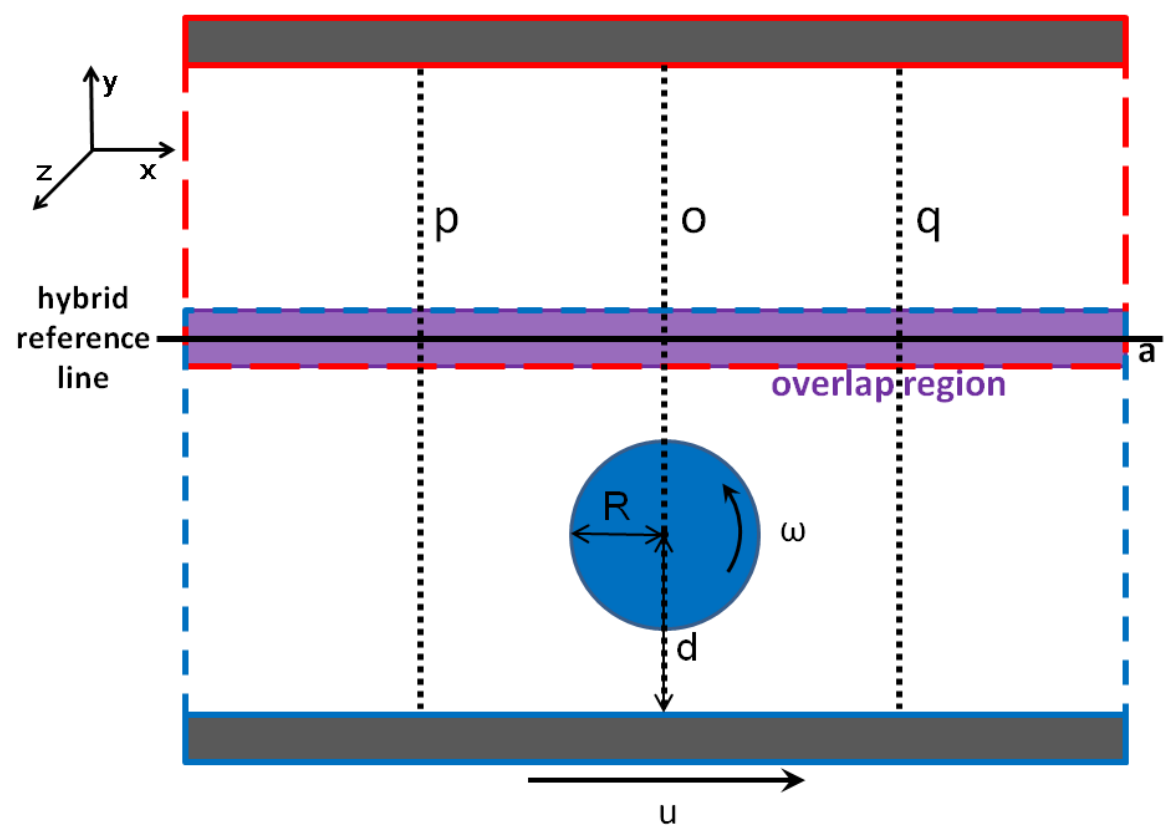

Figure 15: Schematic of the Wannier flow and its domain-decomposition. Sub-domain 1 is the upper part and sub-domain 2 is the lower part. The overlap region is around the hybrid reference line $a$, as explained in Fig. 2, in the middle. Three vertical lines at $x_{o}=L_{x} / 2, x_{p}=L_{x} / 4$ and $x_{q}=3 L_{x} / 4$ are considered as representative locations, where velocity profiles will be evaluated.

\subsection{Wannier-type flow}

In this section, we consider the so-called Wannier flow [21], where a cylinder rotates next to a moving wall. We run a SPH simulation with single high resolution and take its result as the "true" solution. As long as results of DD-SPH simulations agree with the reference simulation, we will consider the coupling algorithm of DD-SPH method validated.

The schematic of Wannier flow and its corresponding domain decomposition are shown in Fig. 15. The cylinder has radius $R=10^{-3} \mathrm{~m}$ and the domain size is $L_{x}=$ $L_{y}=20 R$. The cylinder center is located at $x_{R}=L_{x} / 2$ and $y_{R}=2 R$. The cylinder rotates counter-clockwise at a constant speed of $\omega=9.46 \times 10^{-2} s^{-1}$ and the bottom wall moves to the right with a constant speed of $v_{w}=\omega R$. The top wall stays still, and periodic boundary conditions are applied along $x$-direction. The artificial sound speed is taken as $c_{s}=20 \omega R=1.892 \times 10^{-3} \mathrm{~ms}^{-1}$ and the EOS is taken as Eq. (3b). Therefore, the non-dimensional numbers are $R e=\omega R^{2} / v=0.0946$ and $M_{a}=\omega R / c_{s}=0.05$.

For a single resolution simulation, based on previous experience we need 10 particles along the cylinder radius direction and 10 particles spanning the gap region between cylinder and the wall to have a well resolved fluid flow [22]. Therefore, SPH particles are initially placed on a square lattice with spacing $\Delta x_{1}=R / 10=10^{-4} \mathrm{~m}$, and 


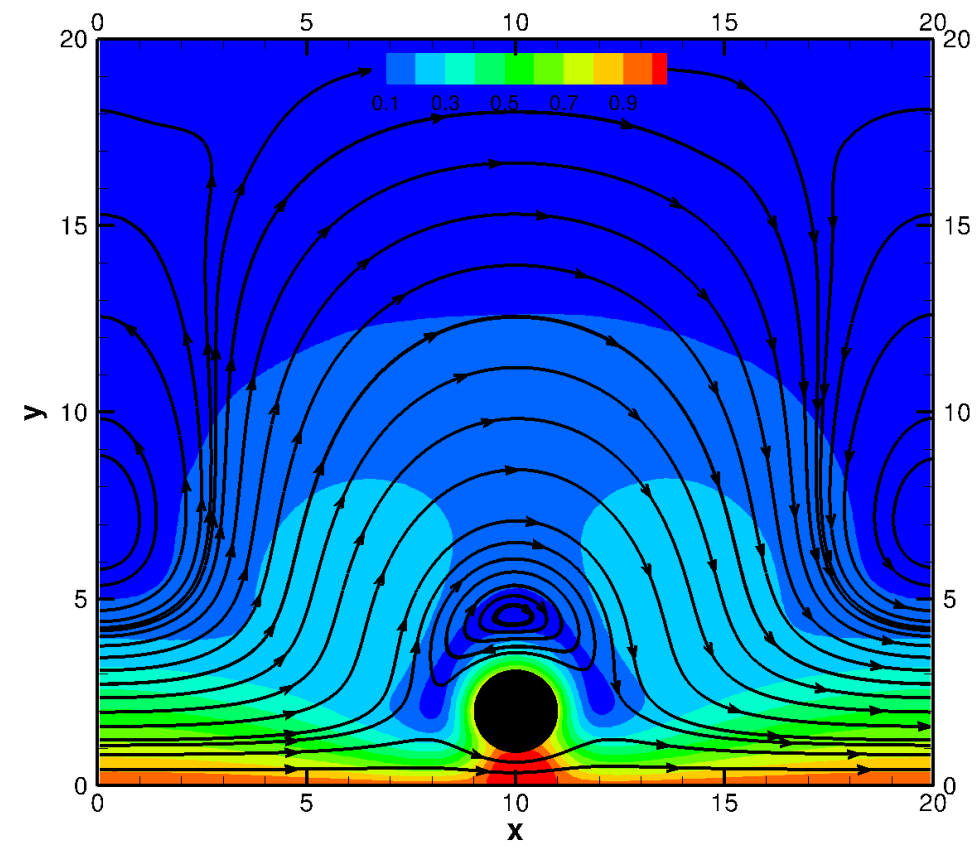

Figure 16: Streamlines and velocity field of Wannier flow by a single resolution SPH simulation. The cylinder has radius $R$ and rotates counter-clockwise with speed $\omega$. Box size is $L_{x}=L_{y}=20 R$ and bottom wall moves with speed $\omega R$ to the right. Reynolds number $R e=\omega R^{2} / v=0.0946$. Color indicates velocity magnitude $\left(v_{x}^{2}+v_{y}^{2}\right)^{1 / 2}$ normalized by $\omega R$.

particle mass $m_{1}=10^{-5} \mathrm{~kg}$ for density $\rho=10^{3} \mathrm{kgm}^{-2}$; the cut off radius $r_{c, 1}=4.4 \Delta x_{1}$ and time step $\Delta t_{1}=2.642 \times 10^{-3} \mathrm{~s}$. The thickness of the walls is taken as the cut off radius. No-slip boundary condition is applied between fluid and solid [14, 22]. The total number of particles for fluid, cylinder and walls is $N_{1}=41504$. We ran the simulation for $10^{6}$ time steps to ensure that the particle configuration becomes disordered and a steady state is achieved. The velocities of particles are averaged in each Eulerian bin of square size $\Delta x_{1}^{2}$ over the last 400 steps. Streamlines drawn according to the bin-averaged velocity field are shown in Fig. 16, which are taken as the reference and will be compared with DD-SPH simulations later on. As Reynolds number is small but above zero, the overall profiles are slightly asymmetric in the $x$ direction.

In the upper part of the domain away from the cylinder, no high resolution is actually needed for the fluid flow, therefore it makes sense to reduce the resolution in the region to save computational cost. In the following, we perform DD-SPH simulations by retaining high resolution in the lower sub-domain (with $m_{1}$ and $\Delta t_{1}$ ) and decreasing resolution in the upper sub-domain (with $m_{2}$ and $\Delta t_{2}$ ). Indeed we have simulated a trivial case with $m_{1}=m_{2}, \Delta t_{1}=\Delta t_{2}$ and a low resolution-ratio case with $m_{2}=4 m_{1}$, $\Delta t_{2}=4 \Delta t_{1}$, but we only show results for a more aggressive and interesting case with $m_{2}=16 m_{1}, \Delta t_{2}=16 \Delta t_{1}$. 


\subsubsection{Hybrid reference line a at $y_{a}=L_{y} / 2=10 R$}

We start with the hybrid reference line $a$ at $y_{a}=L_{y} / 2=10 R$ and build up the overlap region around $y_{a}$ as explained in Fig. 2. Therefore, the lower and upper boundaries of the overlap region are at $y=7.2 R$ and $y=11.4 R$, respectively. In the upper subdomain, we reduce the particle resolution four times in both directions, that is, particles are initially placed on a square lattice with equal spacing of $\Delta x_{2}=4 \Delta x_{1}$. Therefore, $r_{c, 2}=4 r_{c, 1}, m_{2}=16 m_{1}$ and $\Delta t_{2}=16 \Delta t_{1}$. The total number of particles in the lower sub-domain is reduced to $N_{1}=23704$ and the total number of particles in the upper sub-domain is $N_{2}=1850$. The communication time step is taken as $\Delta t_{\text {comm }}=\Delta t_{2}$, and the coupled simulation runs for $t=1.12 \times 10^{6} \Delta t_{1}=7 \times 10^{4} \Delta t_{2}$. The procedure of particle deletion and insertion is performed as described in Section 2.7. More precisely, particles are removed once they leave the artificial boundaries towards the exterior. Inserting locations of new particles are along the exterior edges of the artificial boundaries with equal spacing of $\Delta x_{1}$ in the lower sub-domain and $\Delta x_{2}$ in the upper sub-domain, respectively. The velocities of particles in the lower sub-domain are averaged in each Eulerian bin of square size $\Delta x_{1}^{2}$ over its last 400 steps, while the velocities of particles in the upper sub-domain are averaged in each Eulerian bin of square size $\Delta x_{2}^{2}$ over its last 25 steps. Velocity contours according to the bin-averaged velocity field are shown in Figs. 17a and 17b. Velocity contours (both $v_{x}$ and $v_{y}$ ) in the lower sub-domain from DD-SPH simulation compare very well with the reference simulation. In contrast, velocity contours (especially $v_{x}$ ) in the upper sub-domain from DD-SPH simulation deviate apparently from the reference simulation. The discrepancy is more significant in the left overlap region than in the other regions. Recall the streamlines of the flow, as shown in Fig. 16, the larger deviation takes place at the inflow region of the upper sub-domain. Therefore, it is reasonable to suspect that the particle insertion algorithm with the regular inserting spacing is not accurate and introduces a bias for the flow field. Based on this speculation, we refine the inserting locations of new particles at equal spacing of $\Delta x_{1} / 2$ and $\Delta x_{2} / 2$ for the lower and upper sub-domains, respectively. Results with refined inserting locations are shown in Figs. (17c) and (17d), which represent a significant improvement over the insertion with regular spacings.

\subsubsection{Hybrid reference line a at $y_{a}=8 R$ and $7 R$}

We further move the hybrid reference line $a$ down to be at $y_{a}=8 R$ and $y_{a}=$ $7 R$ and keep the resolution ratio of two sub-domains fixed, that is, $m_{2} / m_{1}=16$ and $\Delta t_{2} / \Delta t_{1}=16$, and perform DD-SPH simulations again. Therefore, for the former case with $y_{a}=8 R$, the overlap region is between $y=5.2 R$ and $y=9.4 R$ and the total particle numbers are $N_{1}=19704$ and $N_{2}=2100$ in the lower and upper sub-domains, respectively. For the latter case with $y_{a}=7 R$, the overlap region is between $y=4.2 R$ and $y=8.4 R$ and the total particle numbers are $N_{1}=17704$ and $N_{2}=2250$ in the lower and upper sub-domains, respectively. Particle inserting locations are with equal spacing $\Delta x_{1} / 2$ for the lower sub-domain and $\Delta x_{2} / 2$ for the upper sub-domain. Velocity profiles of all DD-SPH simulations including the case of $y_{a}=10 R$ are shown in Fig. 18, where the focus is along the three representative vertical lines as illustrated in Fig. 15. Values in the artificial boundaries are removed for clarity and results of two subdomains are pieced together at the hybrid reference line $a$. Data on the right in $\Omega_{2}$ are 


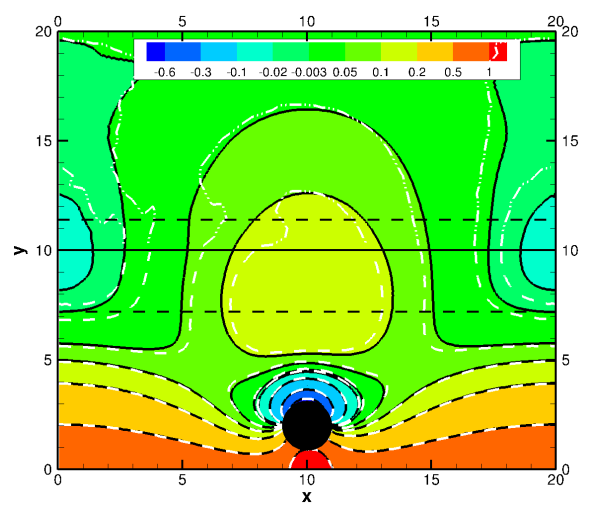

(a) Contour of $v_{x}$.

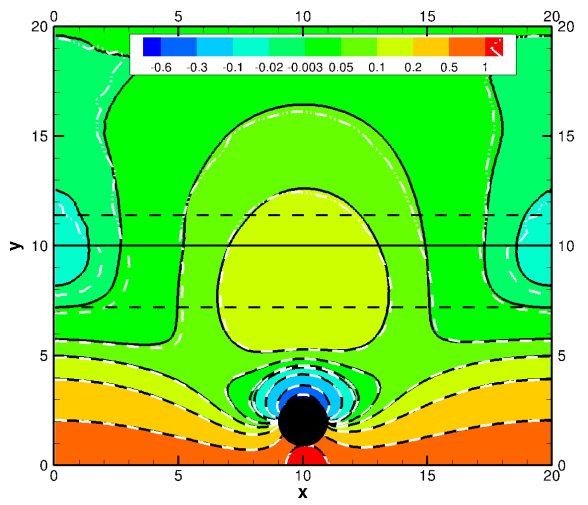

(c) Contour of $v_{x}$ by refined insertion.

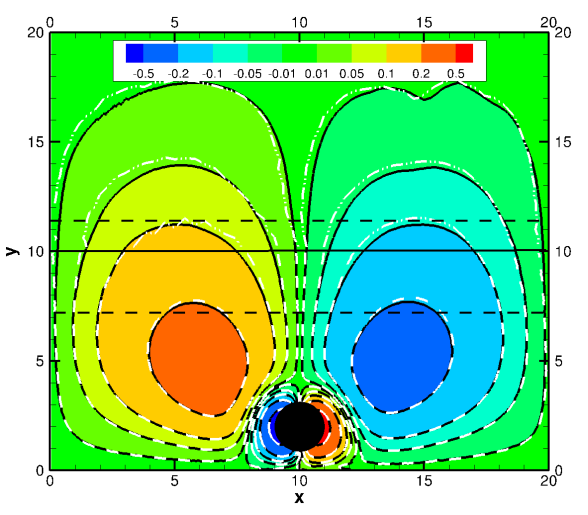

(b) Contour of $v_{y}$.

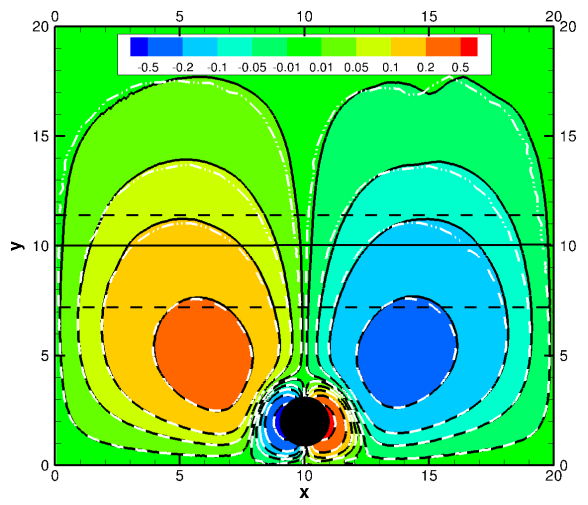

(d) Contour of $v_{y}$ by refined insertion.

Figure 17: Velocity contours of Wannier flow by a domain decomposition based SPH (DD-SPH) method with $m_{2} / m_{1}=16, \Delta t_{2} / \Delta t_{1}=16$ and hybrid reference line at $L_{y} / 2$. Velocity contours of a single high resolution SPH simulation are presented in different colors bordered by solid lines. The rectangular region marked by dashed lines in the middle indicates the overlap region and the horizontal solid line indicates the hybrid reference line. In the DD-SPH simulation, results of sub-domain 1 are below the hybrid reference line (light dash lines) and results of sub-domain 2 are above the hybrid reference line (light dash-dot lines). In Figs. 17a and 17b, particle insertion locations are at regular spacing of $\Delta x_{1}$ and $\Delta x_{2}$ for sub-domain 1 and 2 , respectively. In Figs. $17 \mathrm{c}$ and $17 \mathrm{~d}$, particle insertion locations are at refined spacing of $\Delta x_{1} / 2$ and $\Delta x_{2} / 2$ for sub-domain 1 and 2, respectively. Length is normalized by cylinder radius $R$ and velocity is normalized by its rotating speed $\omega R$. 
sparse due to its low resolution. We observe that results of DD-SPH simulations for $y_{a}=10 R$ match closely the velocity profiles of the reference simulation. Only a small deviation is observed for the cases of $y_{a}=8 R$ and $7 R$ in the overlap region along line $q$, where the overlap region already crosses the vortex above the cylinder.

\section{Summary}

We present a methodology on concurrent coupling of state variables and fluxes of two resolutions of the smoothed particle hydrodynamics (SPH) method. In particular, we decompose a simulation domain into two overlapping sub-domains via the domain decomposition (DD) method. The overlap region consists of two artificial boundary regions and a gap region between them. As the artificial boundary of one sub-domain is embedded in the other sub-domain, constraints are imposed on the density and momentum by Lagrangian interpolation based on information of the other simulation. A conditional rule is applied at the artificial boundaries to correct the conservative force calculation without spherical support and to relax the particle configuration towards the constrained density. Particles are removed once they leave their sub-domain and new particles are inserted at locations with sufficient inward momentum flux accumulated (over a few time steps), as guided by the other simulation. Any newly inserted particle has the corresponding particle mass in the sub-domain and carries the local flow velocity provided by the other simulation. To this end, the global problem is essentially equivalent to two smaller problems in the two sub-domains, yet on each sub-domain we have the flexibility to select a proper resolution in both space and time according to the local flow physics.

We first validated the new coupling method for a transient Couette flow. Based on the analytical solutions, we were able to establish the minimum length scales around the overlap region so that an appropriate thickness of the overlap region enables effective coupling for the transient problem. The overlap region may still be further reduced for a steady problem. From the study on temporal coupling with communication step $\Delta t_{c o m m}$, we observed that if the tolerance on numerical error is not so stringent, we may select $\Delta t_{\text {comm }} \geq \Delta t_{2} \geq \Delta t_{1}$ for a transient problem, where $\Delta t_{1}$ and $\Delta t_{2}$ are time steps for the fine and coarse SPH simulations, respectively. For a steady problem, although any value of $\Delta t_{\text {comm }} \geq \Delta t_{2}$ would not affect the error level, a larger $\Delta t_{\text {comm }}$ seems to slow down the convergence to steady state.

Subsequently, we simulated another type of non-equilibrium problems, where a perturbation wave travels either parallel or perpendicular to the hybrid interface. This type of problem is different from the Couette flow, as there is no external boundary injecting kinetic energy and the wave has a decaying nature. As a consequence, the validity of DD-SPH simulation is completely determined by the correctness of artificial boundary conditions in the overlap region. We showed that the magnitude of momentum and density in the overlap region from the two simulations is equal and the overall numerical solution agrees well with the analytical solution. We further noted that com-

pressibility at short time scale is important and hence we coupled the density fields of the two sub-domains.

Finally, we studied the so-called Wannier flow. In the lower region near the cylinder and its adjacent wall, a high particle number density is required to resolve the lubrica- 


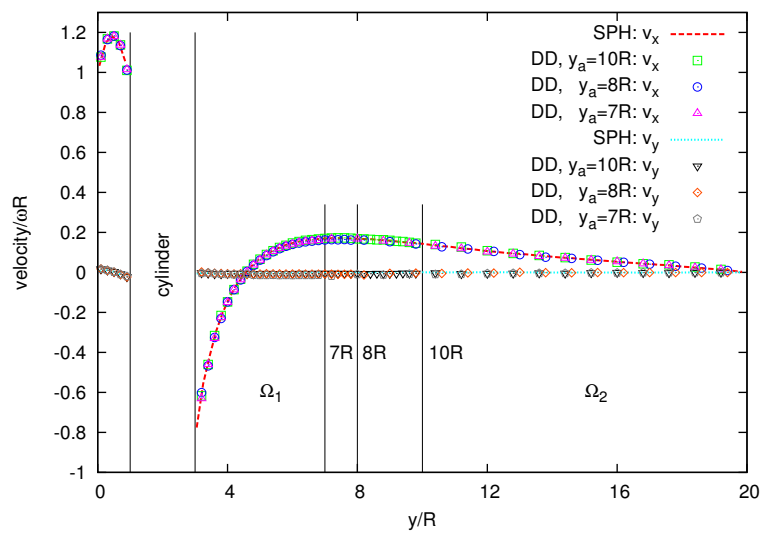

(a) Along vertical line $o$

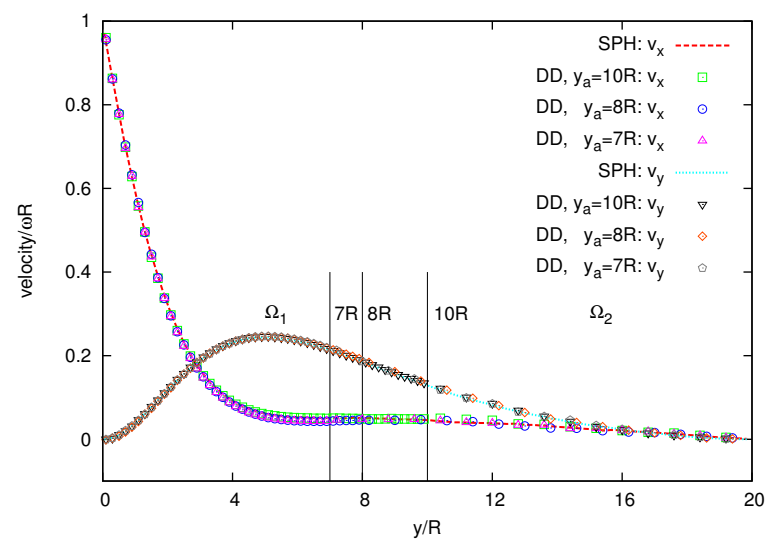

(b) Along vertical line $p$

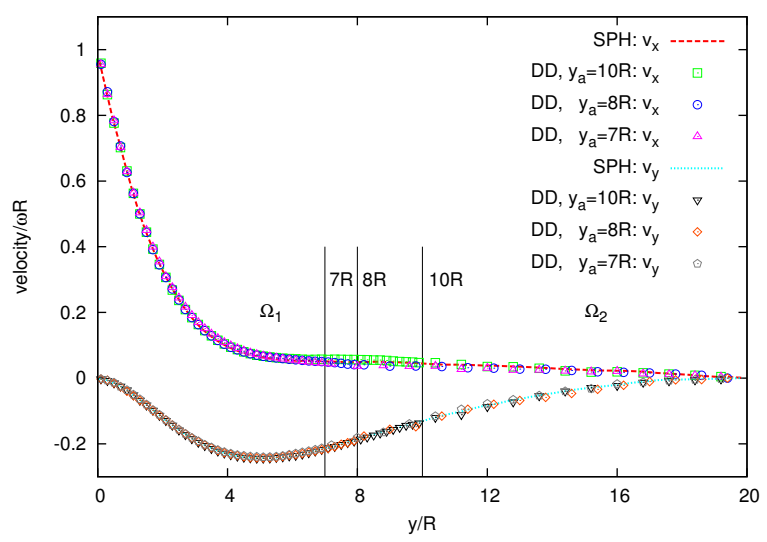

(c) Along vertical line $q$

Figure 18: Velocity profiles at representative lines of Wannier flow. DD stands for domain decomposition based SPH simulations with $m_{2} / m_{1}=16$ and $\Delta t_{2} / \Delta t_{1}=16$. Hybrid reference line $a$ is at $y_{a}=10 R, 8 R$ or $7 R$, as indicated by vertical lines. 
tion gap. To save computational cost, we replaced a single high resolution simulation in the global domain with two smaller simulations in two sub-domains. In particular, we employed high resolution in sub-domain 1 around the cylinder and low resolution in sub-domain 2 away from the cylinder. For the highest mass ratio of two types of particles, we had $m_{2} / m_{1}=16$ and the corresponding time step ratio was $\Delta t_{2} / \Delta t_{1}=16$. Hence, we had approximately a computational speed-up of $16^{2}=256$ for the simulation in the sub-domain 2 for a small additional cost of communication at the artificial boundaries. A further higher mass and time step ratio, and therefore more computational savings would be possible, if the sub-domain 2 was larger. The amount of the additional cost on communication depends strongly on how smart the data structure, distribution of work, and communication pattern are selected in the implementation [23]. For example, the low resolution sub-domain may run on one processor while high resolution sub-domain may run on four processors, where communication takes places between processor 1 and processors $2-5$, in addition to the internal communication within processors $2-5$.

The proposed coupling methodology has strict mass conservation in each subdomain in the case of an incompressible flow simulation, such as the Wannier flow, since any removed particle is inserted back immediately at the location which has the maximum inward momentum flux accumulated (over a few time steps). The momentum conservation is violated locally by the asymmetric calculation of conservative force at artificial boundaries while it is preserved in the rest of the fluid region. However, the results at/near the artificial boundaries are sacrificial and the global result consists of results in the two sub-domains glued together at the hybrid reference line. Therefore, a DD-SPH simulation preserves momentum conservation as a single resolution SPH simulation does.

The proposed coupling methodology avoids intentionally SPH force interactions between two types of particles. Instead, we choose to match the state variables, e.g., velocity and density, in the overlap region with hybrid description. This choice is a key ingredient of the DD-SPH simulations, as this allows for $\Delta t_{2}>\Delta t_{1}$. Furthermore, state variable coupling does not depend on the specifics of the particle method, hence it can be extended in coupling other mesoscopic and microscopic particle methods, such as dissipative particle dynamics (DPD) and molecular dynamics (MD).

In future work, we would address the issues of moving non-planar interfaces, coupling of more adjacent sub-domains, and also coupling of stochastic-deterministic descriptions.

\section{Acknowledgement}

This work was supported by the DOE Collaboratory on Mathematics for Mesoscopic Modeling of Materials (CM4).

\section{Appendix A. Flowchart for the coupling algorithm within LAMMPS code}

We provide a flow chart, as shown in Fig. A.19, for the multi-resolution simulation of SPH via the domain decomposition. In particular, we implemented the particle 


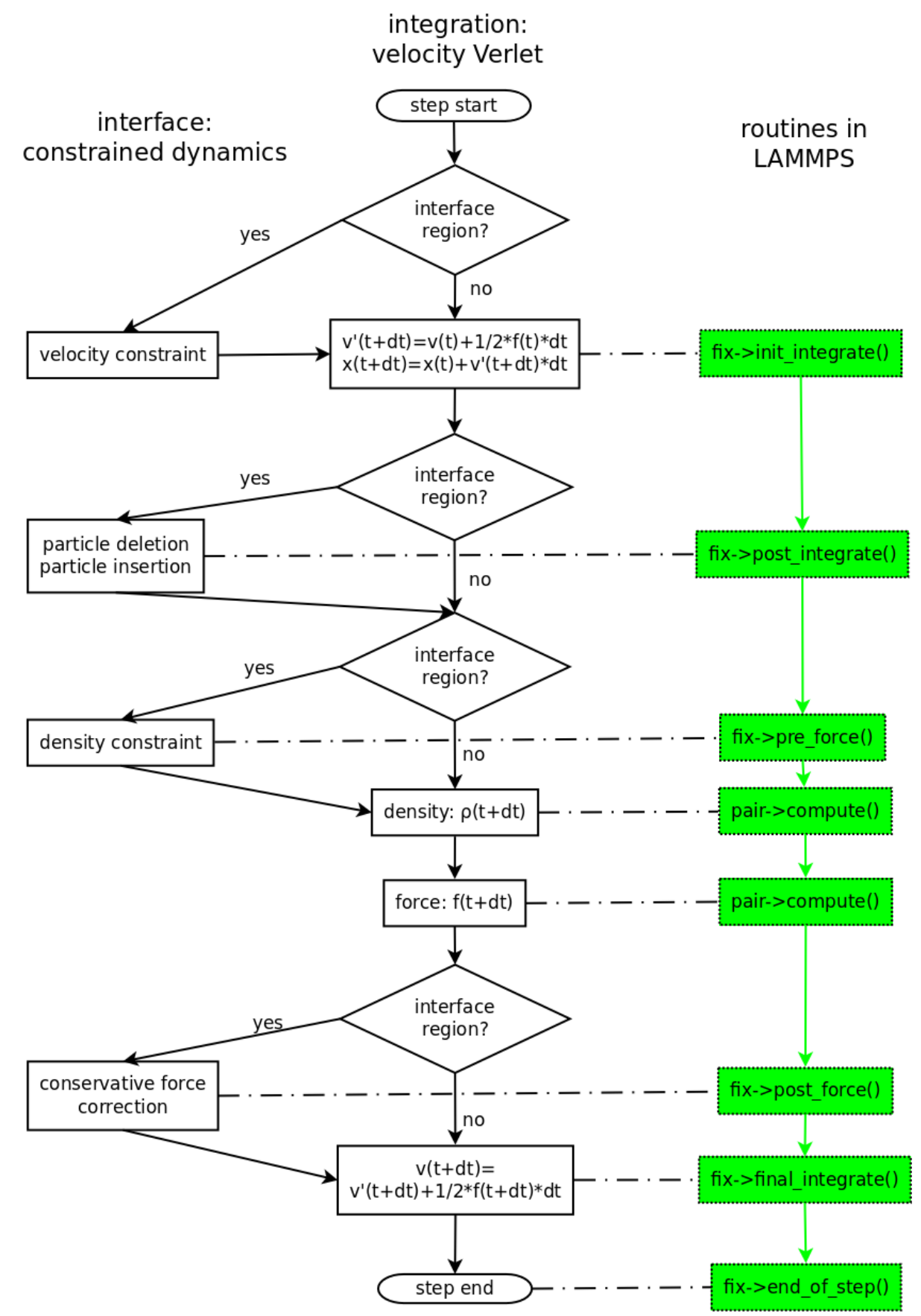

Figure A.19: Flow chart of the algorithm and its corresponding routines in LAMMPS: routines for parallelization are omitted for clarity. 
algorithms and multi-domain coupling within LAMMPS, which is a very popular and fast open source code for particle methods [24]. We show the process flow of one time step of a velocity Verlet integration so that any potential user may implement the coupling algorithm presented. Note that parallelization based on message passing interface is omitted for clarity and readers are referred to the manual of LAMMPS online (http://lammps.sandia.gov) for further technical details.

\section{References}

[1] M. Berger, P. Colella, Local adaptive mesh refinement for shock hydrodynamics, J. Comput. Phys. 82 (1) (1989) $64-84$.

[2] S. T. O'Connell, P. A. Thompson, Molecular dynamics-continuum hybrid computations: a tool for studying complex fluid flows, Phys. Rev. E 52 (1995) R5792 - R5795.

[3] N. G. Hadjiconstantinou, Hybrid atomistic-continuum formulations and the moving contact-line problem, J. Comput. Phys. 154 (2) (1999) $245-265$.

[4] E. G. Flekkøy, G. Wagner, J. Feder, Hybrid model for combined particle and continuum dynamics, Europhys. Lett. 52 (3) (2000) $271-276$.

[5] T. Werder, J. H. Walther, P. Koumoutsakos, Hybrid atomistic-continuum method for the simulation of dense fluid flows, J. Comput. Phys. 205 (2005) $373-390$.

[6] B. Smith, P. Bjorstad, W. Gropp, Domain decomposition: parallel multilevel methods for elliptic partial differential equations, Cambridge University Press, 1996.

[7] J. J. Monaghan, Smoothed particle hydrodynamics, Rep. Prog. Phys. 68 (8) (2005) $1703-1759$.

[8] S. Adami, X. Hu, N. Adams, A transport-velocity formulation for smoothed particle hydrodynamics, J. Comput. Phys. 241 (2013) $292-307$.

[9] X. Bian, S. Litvinov, M. Ellero, N. J. Wagner, Hydrodynamic shear thickening of particulate suspension under confinement, J. Non-Newton Fluid Mech. 213 (2014) $39-49$.

[10] R. P. Nelson, J. C. Papaloizou, Variable smoothing lengths and energy conservation in smoothed particle hydrodynamics, Mon. Not. Roy. Astron. Soc. 270 (1994) $1-20$.

[11] J. Feldman, J. Bonet, Dynamic refinement and boundary contact forces in SPH with applications in fluid flow problems, Int. J. Numer. Meth. Eng. 72 (3) (2007) $295-324$.

[12] D. Barcarolo, D. L. Touzé, G. Oger, F. de Vuyst, Adaptive particle refinement and derefinement applied to the smoothed particle hydrodynamics method, J. Comput. Phys. 273 (2014) 640 - 657. 
[13] G. E. Karniadakis, A. Beskok, N. Aluru, Microflows and nanoflows: fundamentals and simulation, Springer, New York, 2005.

[14] J. P. Morris, P. J. Fox, Y. Zhu, Modeling low Reynolds number incompressible flows using SPH, J. Comput. Phys. 136 (1) (1997) $214-226$.

[15] D. J. Price, Smoothed particle hydrodynamics and magnetohydrodynamics, J. Comput. Phys. 231 (2012) $759-794$.

[16] W. Dehnen, H. Aly, Improving convergence in smoothed particle hydrodynamics simulations without pairing instability, Mon. Not. R. Astron. Soc. 425 (2) (2012) $1068-1082$.

[17] P. Español, M. Revenga, Smoothed dissipative particle dynamics, Phys. Rev. E 67 (2) (2003) 026705.

[18] H. Takeda, S. M. Miyama, M. Sekiya, Numerical simulation of viscous flow by smoothed particle hydrodynamics, Prog. Theor. Phys. 92 (5) (1994) 939-960.

[19] X. Bian, S. Litvinov, R. Qian, M. Ellero, N. A. Adams, Multiscale modeling of particle in suspension with smoothed dissipative particle dynamics, Phys. Fluids (1994-present) 24 (1) 012002.

[20] G. De Fabritiis, M. Serrano, R. Delgado-Buscalioni, P. V. Coveney, Fluctuating hydrodynamic modeling of fluids at the nanoscale, Phys. Rev. E 75 (2007) 026307.

[21] G. E. Karniadakis, S. J. Sherwin, Sepctral/hp element methods for computational fluid dynamics, Numerical mathematics and scientific computation, Oxford University Press, New York, 2nd edn., 2013.

[22] X. Bian, M. Ellero, A splitting integration scheme for the $\{\mathrm{SPH}\}$ simulation of concentrated particle suspensions, Comput. Phys. Commun. 185 (1) (2014) 53 62.

[23] Y.-H. Tang, S. Kudo, X. Bian, Z. Li, G. E. Karniadakis, Multiscale Universal Interface: a concurrent framework for coupling heterogeneous solvers, J. Comput. Phys. (2014) unpublished.

[24] S. Plimpton, Fast parallel algorithms for short-range molecular dynamics, J. Comput. Phys. 117 (1) (1995) $1-19$. 